\title{
Quasielastic Neutron Scattering in Biology: Theory and Applications
}

\author{
Derya Vural $^{(\mathrm{b})}$, Xiaohu Hu${ }^{(\mathrm{b})}$, Benjamin Lindner ${ }^{(\mathrm{a})}$, Nitin Jain ${ }^{(\mathrm{b})}$, \\ Yinglong Miao $^{(\mathrm{b})}$, Xiaolin Cheng ${ }^{(\mathrm{b})}$, Zhuo Liu ${ }^{(\mathrm{a})}$, Liang Hong ${ }^{(\mathrm{a})}$ and Jeremy C. Smith ${ }^{(\mathrm{b})}$
}

a. Institute of Natural Sciences \& Department of Physics and Astronomy, Shanghai Jiao Tong University, China, 200240

b. Center for Molecular Biophysics, Oak Ridge National Laboratory, TN, USA 37831 \& Department of Biochemistry and Cellular and Molecular Biology, University of Tennessee, Knoxville, TN, USA 37996

\section{BBA Special Issue}

\begin{abstract}
$\underline{\text { Abstract }}$
Neutrons scatter quasielastically from stochastic, diffusive processes, such as overdamped vibrations, localized diffusion and transitions between energy minima. In biological systems, such as proteins and membranes, these relaxation processes are of considerable physical interest. We review here recent methodological advances and applications of quasielastic neutron scattering (QENS) in biology, concentrating on the role of molecular dynamics simulation in generating data with which neutron profiles can be unambiguously interpreted. We examine the use of massively-parallel computers in calculating scattering functions, and the application of Markov state modeling. The decomposition of MD-derived neutron dynamic susceptibilities is described, and the use of this in combination with NMR spectroscopy. We discuss dynamics at very long times, including approximations to the infinite time mean-square displacement and nonequilibrium aspects of single-protein dynamics. Finally, we examine how neutron scattering and MD can be combined to provide information on lipid nanodomains.
\end{abstract}

Keywords: Dynamics, Biomolecules, Neutron Scattering, MD simulation

\section{Introduction.}

The dynamics of biomolecular systems, including their associated hydration water, is critical to their folding and function [1-6]. In particular, large-amplitude, collective atomic motions in proteins play a particularly important role in many functional processes, including the entry of substrates into catalytic sites [4], allosteric conformational change [5] and enzymatic reactions [6]. Biomolecular dynamics has been extensively investigated using molecular dynamics (MD) computer simulations $[7,8]$, which historically have been limited to the $p s$ to $n s$ timescales, but now, with specialized hardware, extend out as far as the $m s$ timescale [9]. A result of these simulations is that, even on the $p s$ to $n s$ timescales, biomolecular systems exhibit a wide variety of diffusive and vibrational dynamics $[1,7,8]$. This variety has rendered difficult the derivation from MD of a simplified description of the motions in terms of distinct, superposable components. The corresponding quantitative experimental characterization of motional amplitudes, forms and time dependences is even more challenging. 
Perhaps the most direct experimental technique for determining atomic motions is dynamic neutron scattering, a form of spectroscopy that probes $p s$ and $n s$ atomic motions, as neutrons directly scatter from the position of atoms fluctuating. A large body of research exists on the application of neutron scattering spectroscopy to proteins and other biological systems [e.g., 3,7,8,10-17]. The complementarity of MD to neutron scattering can be exploited to obtain, in principle, a complete description of atomic dynamics. Here, we concentrate on the nonvibrational, diffusive contribution to scattering profiles; the so-called "quasielastic" scattering $[17,18]$, and summarize some recent conceptual and methodological progress made at ORNL concerning the link between motions in MD and experimentally measured scattering.

We review here new concepts in the calculation of quasielastic neutron scattering (QENS) data from MD that permit decomposition of scattering profiles in terms of well-defined collective motions, rather than simply atomic contributions. In this regard we look at the various diffusive dynamical processes awakened in a globular protein as the temperature is increased from zero Kelvins to the physiological regime. We show how MD can decompose the quasielastic dynamic neutron susceptibility into three distinct components, and how surface hydration modifies the local diffusive properties of atoms even in a protein core. We examine the principle of De Gennes Narrowing as applied to inter-domain motions in proteins. De Gennes narrowing is a theory explaining the experimental phenomenon that the coherent diffusion coefficient, $D_{c o h}(q)$, characterizing the fluctuation rate of the interatomic motion, reaches a minimum at the wavevector at which the structure factor, $S(q)$, is a maximum. The underlying mechanism is that the interatomic motion is slowest, i.e., a minimum in $D_{c o h}(q)$, when the system adopts the most probable conformation. This theory has been widely used for explaining the experimental results on inter-particle motion in colloid system, supercooled liquids and biomembranes. We found that the concept also applies for protein inter-domain motion. We also look at the concept of an 'intrinsic' or 'infinite time' mean-square displacement, as derived from neutron scattering amplitudes, and then examine why this may never be reached in vivo, protein dynamics being non-ergodic, non-equilibrium and self-similar even on timescales approaching their lifetimes in the cell. Finally, we look at how neutron scattering and MD can be used to probe lipid raft domains in biological membranes. This review does not pretend to be comprehensive, and we refer readers to Refs. [10,16,19-21] for further, complementary information.

\section{Calculating QENS}

Incoherent neutron scattering is expressed in terms of a time correlation function, the intermediate scattering function (ISF), $I(q, t)$, given by,

$$
I(\vec{q}, t)=\frac{1}{Z} \sum_{j}^{Z} b_{i n c, j}{ }^{2}\left\langle\exp \left[-i \vec{q} \cdot \vec{r}_{j}(0)\right] \exp \left[i \vec{q} \cdot \vec{r}_{j}(t)\right]\right\rangle
$$

where $q$ is the scattering wavevector, $Z$ the total number of the hydrogen atoms, $b_{i n c, j}$ the incoherent scattering length of atom $j, r_{j}$ the position vector of that atom and the brackets denote an ensemble average. The coherent ISF is given by 


$$
I_{c o h}(\vec{q}, t)=\sum_{i}^{Z} \sum_{j}^{Z} b_{c o h, i} b_{c o h, j}\left\langle\exp \left[-i \vec{q} \cdot \vec{r}_{i}(0)\right] \exp \left[i \vec{q} \cdot \vec{r}_{j}(t)\right]\right\rangle,
$$

where $b_{c o h, j}$ is the coherent scattering length. Thus, incoherent scattering is determined by selfcorrelations (particularly, due to $b_{i n c, j}$, hydrogens) and coherent scattering has a contribution from cross-correlations.

The dynamic structure factor (DSF) for either incoherent or coherent scattering, is the Fourier transform of the $I(q, t)$, given by

$$
S(\vec{q}, \omega)=\frac{1}{2 \pi} \int_{-\infty}^{\infty} d t \exp (i \omega t) I(\vec{q}, t)
$$

where $\omega$ is the energy transfer between the neutron and the sample.

In recent years, the ability to calculate and interpret QENS directly from MD trajectories has been enhanced through the following two advances: the development of optimized software for the calculation of QENS on massively parallel supercomputers, and the use of Markov State Theory to model QENS data for large time scales.

The backbone of the calculation of QENS from MD is software to calculate scattering functions from atomic trajectories. Several different special purpose software programs have been written for this purpose, including the popular software packages nMoldyn [22], Serena [23], SASSIM [24] and CRYSOL [25]. All these software packages have in common the calculation of atomic scattering amplitudes $a_{j}(\vec{q}, t)=b_{j} \exp \left[-i \vec{q} \vec{r}_{j}(t)\right]$ including a pre-factor, distinguishing X-ray from neutron scattering. The $b_{j}$ pre-factor is isotope dependent. For X-rays the pre-factor is additionally q-vector dependent. The purpose of the software is then to compute and process the atomic scattering amplitudes as efficiently as possible.

Many software packages have been designed for serial computers and thus do not scale well on parallel computers. This limits their applicability to small molecules or sample sets, or makes data aggregation tedious. Recent effort has been made to port some software packages [26] for the use of parallel computers.

Sassena has been developed as a universal software package for calculating scattering functions, focusing on the core task of computing static and dynamic scattering signals efficiently on high performance parallel computers [27]. Available free of charge [http://www.sassena.org], it integrates the core functionality offered by most available software for computing scattering signals, such as multi-dimensional scattering diagrams [23], background-corrected small angle neutron scattering (SANS) [24], isotropic and anisotropic orientational averaging, and coherent and incoherent ISFs [22]. Sassena has been shown to scale well on massively-parallel computers for all classes of scattering functions and has a modular calculation scheme, allowing it to calculate different experimentally relevant functions like elastic incoherent structure factor (EISF), QENS, SANS while maintaining data allocation patterns and algorithmic flow, leading to more consistent and predictable software performance. Sassena is currently being further developed for the integration with GPUs for performance gains 
and integration with additional methodologies like Markov Model State Theory, as now discussed.

A key problem in the interpretation of QENS has been the decomposition of the scattering function into a number of well-defined and superposed relaxation processes. Hitherto, analytical models have been employed that represent quasielastic scattering as a sum of Lorentzians. However, decomposition of detailed MD into superposable elements in non-trivial. Recent advances involve the use of Markov State Theory, which models long-time relaxation processes in terms of a network of transitions between metastable states [28-30], associated with a set of characteristic relaxation times for transitions between the states. The particular procedure by which a Markov Model is determined allows the analysis of the MD to be fragmented into short simulations each spanning only particular transitions [31,32]. This approach is highly scalable and makes the long-time dynamics of proteins attainable with the use of large supercomputing facilities [http://www.olcf.ornl.gov] or even distributed computing networks such as folding@home [http://folding.stanford.edu].

It has been shown that Markov State Modeling can be used to conveniently interpret general experimental correlation functions in terms of structurally well-defined transitions between specific states [33], and this work was recently extended to the calculation of neutron and X-ray scattering signals from Markov Models describing MD simulations [34]. A companion work [35] to Ref. [34] illustrated the successful decomposition of the dynamics of a model system, the alanine dipeptide, into a set of eigen-processes with characteristic eigenvectors and associated relaxation times.

The most significant change in the calculation procedure of neutron and X-ray scattering functions using Markov Models is the change from a time-representation towards a staterepresentation of the system. The incoherent ISF is then represented as linear composition of Markov processes with exponential decay factors, $\exp \left(-\kappa_{j} \tau\right)$ :

$$
I_{i n c}(\vec{q}, \tau)=\sum_{j} \exp \left(-\kappa_{j} \tau\right) \cdot A_{j}(\vec{q})
$$

The information about the time and any relaxation is implicitly preserved in the transition kernel of the Markov Model. The scattering amplitude, $A_{j}(\vec{q})$, is fundamentally derived from the state represented individual atomic scattering amplitudes defined as:

$$
\left\langle a_{j}(\vec{q}, m)\right\rangle_{t}=\left\langle\delta(m-s(t)) \cdot b_{j} \exp \left[-i \vec{q} \vec{r}_{j}(t)\right]\right\rangle_{t}
$$

where $s(t)$ is a function representing the assigned Markov state, $m$, for a given time and $\delta(m-s(t))$ selects for time averaging only those atomic scattering amplitudes which contribute to the expectation value of Markov State $m$. The state-represented atomic scattering amplitudes $\left\langle a_{j}(\vec{q}, m)\right\rangle_{t}$ for all Markov states are then used to provide a weight to the Markov transition kernel eigenvectors, leading to neutron or X-ray scattering dependent observables. These work both for incoherent and coherent scattering [35]. Fig. 1 provides a Markov model staterepresentation for the alanine-dipeptide. 


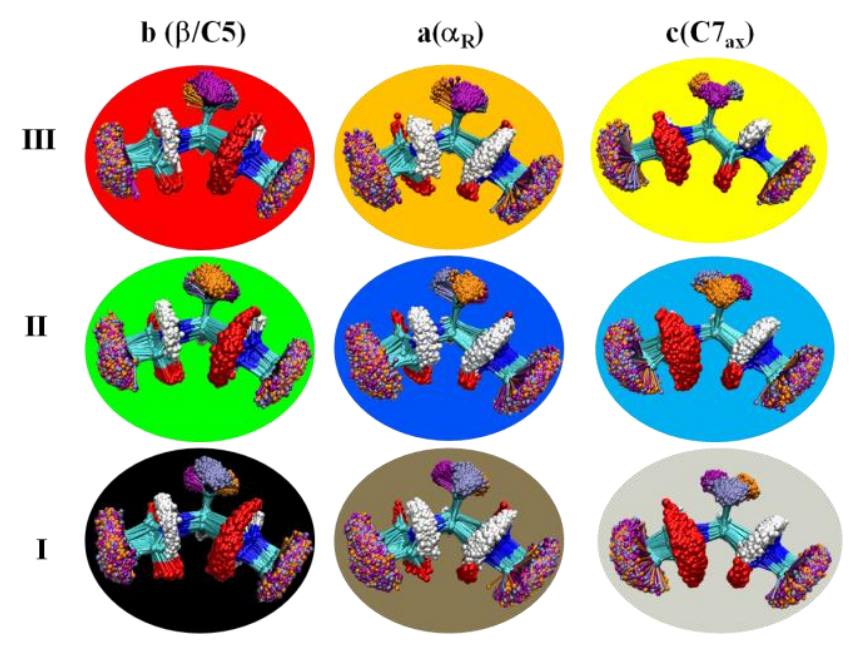

Figure 1 Markov model state representation of alanine dipeptide. The configurational freedom within each Markov state causes the positions of individual atoms to transform into a probability density, represented with the help of individual atom colors. Since the terminal methyl groups were not used in the Markov decomposition, the corresponding atoms occupy all possible methyl group rotation angles. From Ref. [35]

The correlation function derived from the Markov Model is immediately provided as separate components of the Markov eigenvector decomposition. This has a few advantages. For example, the component based on the eigenvector with the eigenvalue of 1 (corresponding to infinite relaxation time), which yields the equilibrium distribution of the simulated system, directly yields the elastic structure factor (ESF). Furthermore the time independent contribution, $A_{j}(\vec{q})$ directly signifies the importance of the mode in the overall scattering function, with dominant modes having the largest values for $A_{j}(\vec{q})$, as is illustrated in Fig. 2.

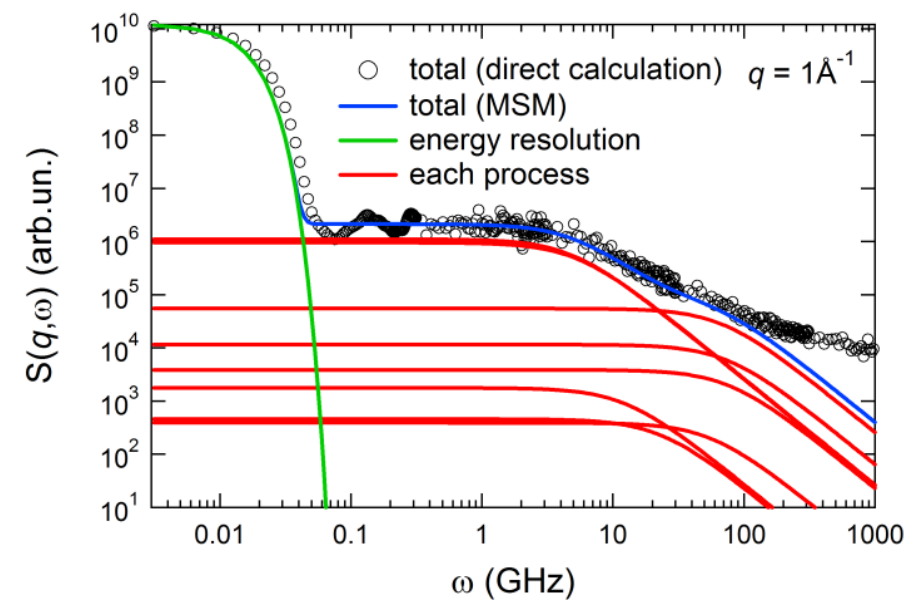

Figure 2 Incoherent dynamic structure factor for alanine dipeptide $S_{\text {inc }}(q, \omega)$ at $q=1 \AA^{-1}$. Dotted lines represent $S_{\text {inc }}(q, \omega)$ calculated from a time representation, solid blue line from the corresponding Markov state model. The figure contains additional lines representing the decomposition into the different Markov modes. From Ref. [35]. 


\section{Three Classes of Motion}

The above methodology, which describes QENS in terms of Markov State processes, has not yet been applied to interpret experimental scattering from proteins. However, decomposition of MD-derived QENS signals is still possible if QENS spectra are presented as the imaginary part of the dynamic susceptibility, $\chi^{\prime \prime}(q, v) \propto \frac{S(q, v)}{n_{B}(v)}$, where $n_{B}(v)$ is the Bose occupation number $n_{B}(v)=(\exp (h v / k T)-1)^{-1}$, and $v=\omega / 2 \pi$. Whereas relaxation processes on different time scales are usually hard to identify in $S(q, v)$, they appear as distinct peaks in $\chi^{\prime \prime}(q, v)$ with associated relaxation times $\tau=1 / 2 \pi \nu[8]$.

In Ref. [36], it was shown that the dynamic neutron spectra of hydrated lysozyme measured experimentally and derived from MD are in quantitative agreement over a wide frequency range (1-1000 GHz). Using the dynamic susceptibility representation, analysis of the MD enabled the microscopic decomposition of the complex protein atomic motions into three simple, distinct classes: "localized diffusion," "methyl group rotations," and "non-methyl jumps". These three types of motions exhibit distinct features on their neutron susceptibility signals (Fig. 3). These distinct and separable features can be utilized to distinguish between changes in dynamical phenomena accompanying biological function.
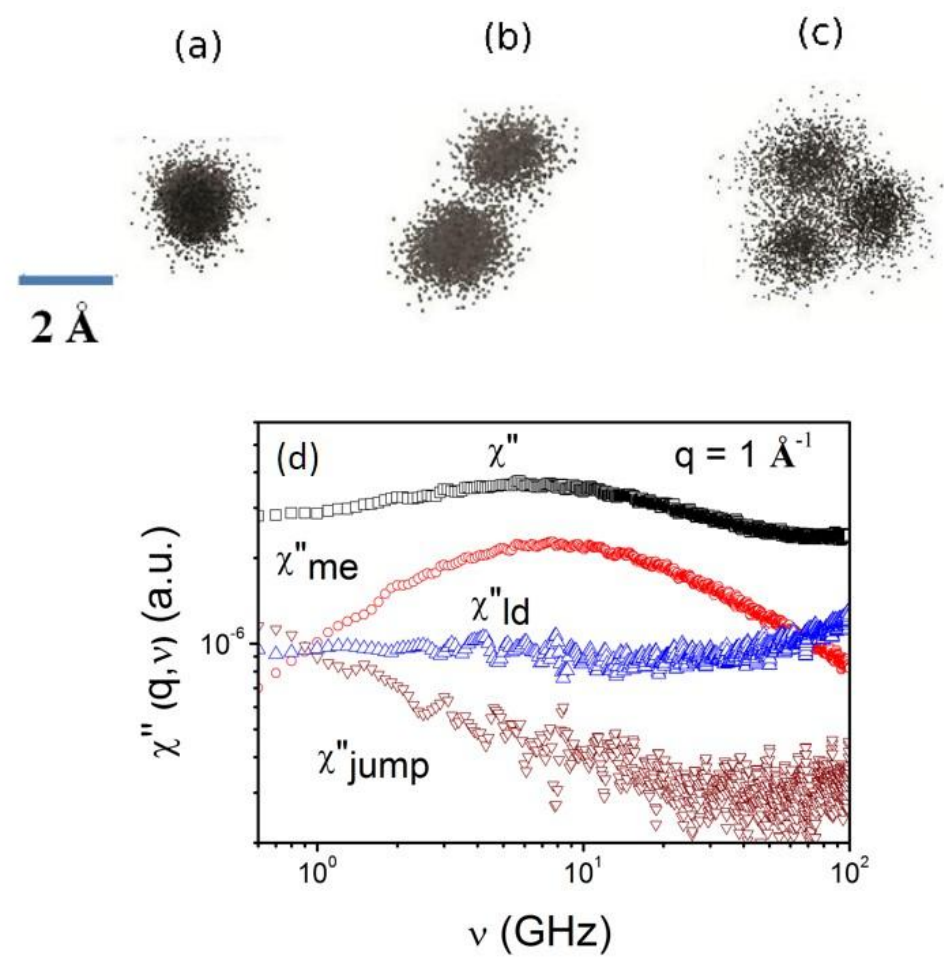

Figure 3 Typical examples of scatter plots of nonexchangeable hydrogen atoms. (a) Localized diffusion (single-well motion) (atom HA of residue ALA37). (b) Two site jumps (atom HD1 of residue ARG 73). (c) Methyl group rotation (atom HB1 of residue ALA 37). (d) MD neutron susceptibility spectrum of the overall protein dynamics $\left(\chi^{\prime \prime}\right)$, the localized diffusion $\left(\chi^{\prime \prime d}\right)$, the methyl group rotations $\left(\chi_{\text {me }}\right)$, and the jumps $\left(\chi_{\text {jump }}\right)$. Each scatter plot was made by projecting the $10 \mathrm{~ns}$ trajectory of a given hydrogen atom at each pico-second into a single graph. From Ref. [36] 


\section{Hydration}

The dynamic susceptibility representation is also useful for examining environmental effects on biomolecular relaxation process, such as hydration. At physiological temperatures, hydration enhances diffusive motions of atoms in protein molecules [8,14,37,38], and this leads to the intriguing question as to whether the hydration effect is mainly local and limited to the protein surface, or rather is global, propagating into the functional core, and if the latter, what the mechanism of the propagation might be NMR and fluorescence anisotropy experiments have provided some evidence that hydration indeed significantly promotes $p s-n s$ atomic motions in the protein interior [39-41]. However, proteins exhibit a wide variety of diffusive and vibrational dynamics on these time scales $[8,14,36,42]$, and most experimental techniques provide only averaged information, rendering difficult the characterization of the microscopic nature of the hydration-promoted atomic motions and of the mechanisms by which the dynamical hydration effects may propagate from the surface into the core.

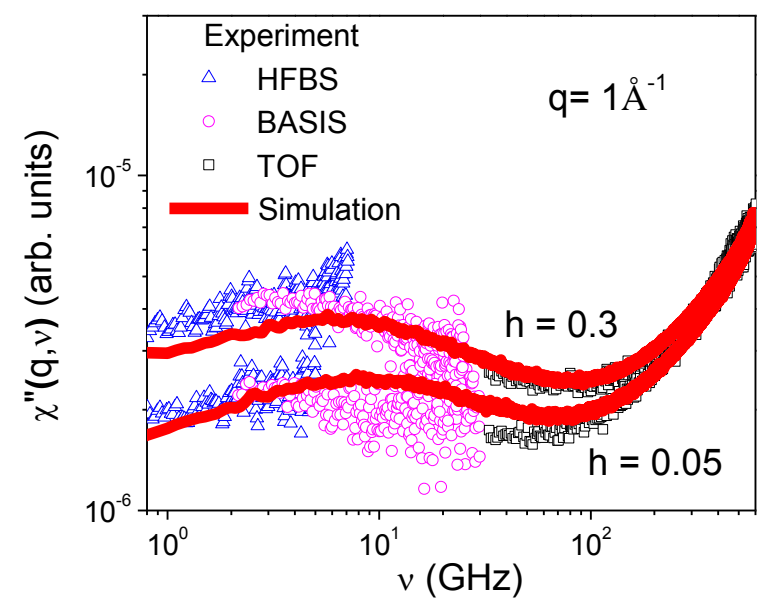

Figure 4 Hydration dependence of the susceptibility spectra of lysozyme $\left(q=1 \AA^{-1}\right)$ at $295 \mathrm{~K}$ obtained from neutron scattering experiments on the NIST high-flux backscattering spectrometer (HFBS, triangles), the ORNL SNS back-scattering spectrometer (BASIS, spheres), and the NIST disk-chopper time-of-flight spectrometer (TOF, squares) $[8,43,44]$ and from MD simulation (thick solid line). To improve the statistics, the experimental spectra were summed over the $q$ range from 0.3 to $1.7 \AA^{-1}$ with an average $q=$ $1 \AA^{-1}$. As the statistics of the simulation-derived spectra are much better, only data at $q=1 \AA^{-1}$ are presented. For ease of comparison of the two sets of spectra, the experimental data on the y axis have been rescaled by a same constant. Experimental details can be found in Ref. [43].

In Fig. $4, \chi^{\prime \prime}$ at different hydration levels derived from MD simulation is compared with the corresponding experimental data, which were obtained by combining results from three instruments $[8,16,43]$. The simulation-derived spectrum agrees quantitatively with the experimental result in a wide frequency window. $\chi$ " shows a broad peak, the so-called "main" relaxation peak [36], covering the $\sim 1$ to $100 \mathrm{GHz}$ frequency range, corresponding to atomic motions on the time scales of a few to hundreds of ps. Using the decomposition method discussed above, the effect of surface hydration water on protein internal dynamics was explored. The analyses revealed that the effect of surface hydration is mainly to increase the volume of the 
localized single-well diffusion and that these diffusive motions are coupled in such a way that the hydration effect propagates from the protein surface into the dry core [45]. This work clearly illustrates the usefulness of combining MD with QENS in the determination of biomolecular dynamical responses.

Another way of looking at internal protein motions is to realize that dynamics can be decomposed into two components, one 'conformational', involving jumps between potential wells, and the other 'elastic', corresponding to fluctuations within the wells. Roughly speaking the conformational component would lead to QENS, whereas the elastic component would lead to inelastic scattering. However, the experimental separation of the contributions of these two components of macromolecular flexibility is challenging. This difficulty has recently been overcome by a combination of QENS, light scattering and MD simulation [46]. The curvature of the potential of mean force for single-well atomic motions is determined by the elastic modulus, which can be determined using light scattering, while the conformational component can be estimated by subtracting the elastic neutron scattering from the overall mean-squared atomic displacement, measured using incoherent neutron scattering. Therefore, a method now exists providing an experimental means of dissecting the conformational from the elastic contribution to flexibility of bio-macromolecules [46]. Again, this may prove useful in the interpretation of dynamical responses as measured by QENS.

\section{Coherent Scattering}

Until recently, most dynamic neutron scattering work on protein was performed using incoherent scattering, thus focusing on analyzing the self-motions of individual hydrogen atoms using hydrogenated proteins. However, coherent scattering, which provides information directly on the collective nature of motions, promises to be of particular biological relevance. In physiological conditions, functional dynamic modes typically occur on a length scale of a few $n m$ and time scales of $n s-\mu s$ or longer $[3,47,48]$. They are a form of QENS because they are typically diffusive in nature. Motions on these length- and timescales are accessible to neutron spin echo spectroscopy (NSE), and this technique has recently been used to probe relative domain motions in several proteins $[3,47,49,50]$. In contrast to other neutron quasi-elastic and inelastic techniques, NSE measures the coherent ISF, rather than the incoherent DSF.

NSE provides direct information on a key spatiotemporal property of domain motion the distance-dependence of the relative motion of protein domains - characterized by the wavevector $(q)$ dependence of the effective diffusion coefficient, $D_{\text {eff }}(q)[3,47,49]$. $D_{\text {eff }}(q)$ is obtained from the initial decay rate of the NSE-determined coherent ISF, given by $-\frac{1}{q^{2}} \lim _{t \rightarrow 0} \frac{d}{d t} \ln \left[\frac{I_{c o h}(q, t)}{I_{c o h}(q, 0)}\right]$.

Recent work analyzing NSE and MD from a variety of protein systems demonstrated that protein inter-domain motion follows the principle of "De Gennes Narrowing", i.e., that $D_{\text {eff }}(q)$ possesses an inverse $q$ dependence on the inter-domain structure factor (Fig. 5). $D_{\text {eff }}(q) \sim 1 / S(q)$ has been observed in many non-biological systems, ranging from liquid argon [51] and deuterated methane [52] through to colloidal particles [53-55]. 
A description of why De Gennes narrowing is seen can be obtained by assuming that two particles, separated by a distance $r$, move relative to each other, and that the associated distance distribution, $P(r)$, peaks at $r^{*}$. Fourier transform of $P(r)$ from real to reciprocal space results in the inter-particle structure factor, $S(q)$ peaking at $q^{*}\left(q^{*} \sim 1 / r^{*}\right)$. Accordingly, the underlying free energy profile exhibits a minimum at $r^{*}$. De Gennes Narrowing predicts that the rate of the interparticle fluctuation is a minimum when the population of the configuration is a maximum, i.e., at $r^{*}[56,57]$. As $D_{\text {inter }}(q)$ is an estimate of the inter-particle fluctuation rate at the distance $r(r \sim$ $1 / q)$ [56,57], it will exhibit a minimum at $q^{*}\left(q^{*} \sim 1 / r^{*}\right)$ where the fluctuation rate reaches a minimum, and thus resembles $1 / S(q)$. Thus, the rate of interdomain motion is inversely proportional to the probability distribution of the spatial configurations of domains. Domains move slower with respect to each other when in a favored spatial arrangements [58]. Fig. 5 demonstrates De Gennes Narrowing in the NSE spectrum of an enzyme. This would suggest that the neutron NSE spectrum can be obtained by simple inversion of the SANS profile. However, while this may hold for certain timescales, at longer timescales there may well be deviation from the De Gennes relation, and these deviations promise to be of particular interest for the future.

(a)

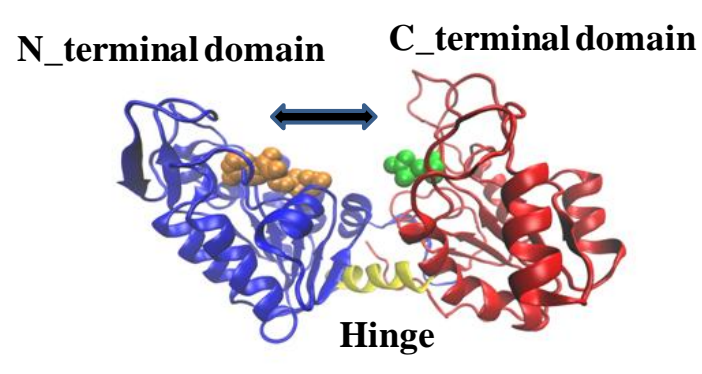

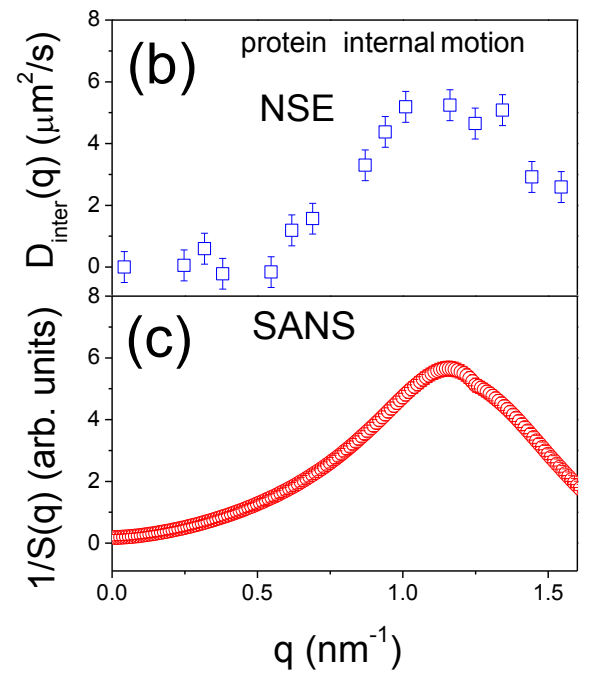

Figure 5 (a) Structure of phosphoglycerate kinase (PGK) consisting of two domains (left and right) of almost equal mass connected by a helix (middle). (b) Effective diffusion coefficient for internal motions of PGK, in aqueous solution collected using NSE [47]. (c) Inter-domain structure factor, $S(q)=I(q) /\left(\sum F_{i}\right.$ $(q))$, where the form factor of the whole protein molecule, $I(q)$, was measured by SANS [47] and that for each single domain, $F_{i}(q)$, was calculated based on the crystal structure (PDB entry 3PGK [59]).

\section{Combination of neutron scattering with MD and NMR for functional motions}

We now consider the combination of QENS and MD with other techniques in a 'multiprobe' approach. The inherent value of neutron scattering as a biophysical technique is greatly increased when it can be utilized for in-depth characterization of protein internal dynamics in combination with other powerful experimental methods for measuring protein dynamics such as NMR spectroscopy. Neutron scattering and NMR spectroscopy both provide insight into protein dynamics, albeit with differing dynamic resolution; whereas neutron scattering provides information on global flexibility of a protein, solution NMR has the potential of measuring 
dynamics at an atomic level on a timescale ranging from picoseconds to seconds [60]. The motional timescales measured by the two techniques overlap in a complementary way in the fast motional regime, where inelastic and quasi-elastic neutron scattering methods can be used to probe atomic motions on the $10^{-1}$ to $10^{4}$ ps timescale, while NMR spin relaxation experiments can provide detailed residue-specific information on fast internal protein dynamics on the $p s-n s$ timescale. Neutron scattering, more importantly, can augment the local level information on this timescale by characterizing the dynamical amplitudes and coupling of the various regions involved in short-timescale ( $p s-n s$ ) diffusive motions, which are difficult to obtain by NMR spin relaxation methods.

Direct physical interpretation of the experimental data from both neutron scattering and NMR in a coherent manner is possible via MD simulations as contributions of the neutron scattering spectra can be decomposed [19], as discussed above, while agreement with the corresponding NMR dynamic data can be obtained in a quantitative manner in terms of residuespecific backbone and side-chain order parameters. The inclusion of neutron scattering and MD simulation data for the visualization of overall protein internal dynamics is particularly useful in situations when NMR data are sparse, particularly for side-chains, a situation often encountered for large proteins due to lack of sufficient resonance assignments. At the same time, availability of a small amount of NMR data can reasonably validate MD simulation trajectories at a local level reasonably possible, overcoming some of the limitations of MD in terms of inaccurate force-fields and model approximations. The synergistic use of the spectroscopic and simulation techniques in this manner allows a more complete and reliable physical picture to be obtained of both the global structural flexibility and local fluctuations in the protein.

A recent application of the combination of neutron scattering, NMR and MD targeted the hemeprotein monooxygenase superfamily of cytochrome P450s, which catalyze the insertion of oxygen atoms in unreactive $\mathrm{C}-\mathrm{H}$ bonds of organic substrates. $\mathrm{P} 450$ enzymes are of high relevance in this regard due to their participation in a variety of chemical reactions including drug metabolism, steroid biosynthesis, degradation of xenobiotic compounds such as environmental pollutants and potential biotechnological applications involving synthesis of unnatural, synthetic substrates. In order for P450s to perform these functions, their structures have to be highly flexible to recognize substrates with diverse physicochemical properties and adapt their active sites for stereo- and regio-selective monooxygenase chemistry. Given their broad substrate specificity, there is great interest in characterizing the inherent conformational flexibility of P450s, which can be crucial information in deciding whether a certain P450 will bind to a given substrate, increasing the efficacy of drug design methods or for rational bioengineering of improved P450 biocatalysts for potential application in the chemical industry.

QENS was applied in the P450 study to elucidate the nature and extent of dynamic changes upon binding of a substrate in a model P450 system cytochrome P450cam (CYP101) [61]. CYP101 is known to have structural flexibility in several regions such as the B' helix, F/G loop and $\beta 5$ sheet which comprise part of a putative substrate access channel undergoing dynamic opening and closing motions that can allow access to a substrate [62] (Fig. 6a). In order to characterize these dynamic motions, QENS measurements were carried out on hydrogenated CYP101 samples in both substrate-free and substrate (camphor) -bound forms at a hydration level of $\mathrm{h}=0.4 \mathrm{~g} \mathrm{D}_{2} \mathrm{O} / \mathrm{g}$ protein using three instruments with different energy resolutions - the NG2 high-flux backscattering spectrometer (HFBS) with an energy resolution of $1 \mu \mathrm{eV}$ at the 
Center for Neutron Research at National Institute of Standards and Technology, the Backscattering Spectrometer (BASIS) with an energy resolution of $3.5 \mu \mathrm{eV}$ [63] and the Cold Neutron Chopper Spectrometer (CNCS) with an energy resolution of $25 \mu \mathrm{eV}$ [64] at the Spallation Neutron Source (SNS) in Oak Ridge National Laboratory.

Since the instruments cover a broad energy range from $1 \mu \mathrm{eV}$ to $1.6 \mathrm{meV}$, it was possible to combine the dynamic susceptibility $\chi^{\prime \prime}(q, v)$ from the three instruments to derive a distinct spectral peak and directly obtain the maximum relaxation amplitude $\left(v_{\max }\right)$ from the peak along with the associated relaxation time, $\tau$ given by $\left(2 \pi v_{\max }\right)^{-1}$. Both substrate-free and camphor-bound CYP101 show broad peaks centered around $2 \pi v_{\max } \sim 3.5 \mathrm{GHz}(\mathrm{E}=14.5 \mu \mathrm{eV})$ and similar relaxation times of $\tau=45 \mathrm{ps}$, however the substrate-free form showed higher intensity with the integral of $\chi^{\prime \prime}(q, v)$ over the quasielastic energy range $(2-200 \mu \mathrm{eV})$ about $5 \%$ greater than the camphor-bound form (Fig. 6b). This indicates that on the $\sim 10^{-1}-10^{3}$ ps time- and $\sim 1-10 \AA$ length-scales, both substrate-free and camphor-bound CYP101 exhibit diffusive dynamics spread over similar relaxation times, but that the substrate-free form undergoes larger amplitude motions than does the camphor-bound form.

Molecular dynamics (MD) simulations of $\sim 100$ ns length were utilized to interpret the neutron scattering data [19]. The dynamic susceptibility values $\chi^{\prime \prime}(q, v)$ calculated from MD simulations for both forms were found to be similar with peaks at a comparable frequency of $2 \pi v_{\max } \approx 3.2 \mathrm{GHz}(\tau \approx 50 \mathrm{ps})$ as the experiment, and the substrate-free form showing largeramplitude diffusive dynamics than the camphor-bound form (Fig. 6c). These studies thus clearly showed the significant value afforded by neutron scattering in comparing the global dynamics of an enzyme as a function of substrate binding.

As well as the neutron scattering measurements and MD described above, NMR spin relaxation studies were performed on ${ }^{15} \mathrm{~N}$ labeled samples of substrate-free and camphor-bound CYP101 to measure longitudinal relaxation time constant $\left(T_{1}\right)$, transverse relaxation time constant $\left(\mathrm{T}_{2}\right)$ and Nuclear Overhauser Effect (NOE) difference intensities, which were then analyzed to extract dynamic differences in form of residue-specific order parameters $\left(\mathrm{S}^{2}\right)$ using a model-free formalism [65,66] (Fig.7a). Order parameters were also calculated from long timescale $(\sim 0.4 \mu \mathrm{s})$ all-atom MD simulations and were found to reproduce the experimental data quite well [61] (Fig. 7b). Both techniques show that, in general, substrate-free CYP101 exhibits lower values of order parameters, indicating substrate-free CYP101 is more dynamic than camphor-bound CYP101 on all time-scales accessible to these experiments, similar to the observations from the neutron scattering experiments. The largest dynamic differences between the substrate-free and camphor-bound forms are seen for residues in the B' helix, portions of $\mathrm{C}$ helix and I helix as well as the BC-and FG-loops, indicating the importance of these regions in substrate binding.

The quasielastic neutron scattering spectra were decomposed in terms of the contribution of the five different protein regions that show the largest dynamic differences between the substrate-free and camphor-bound CYP101 based on the NMR-derived order parameters (the $\mathrm{B} / \mathrm{C}$ and $\mathrm{F} / \mathrm{G}$ loops, the I-helix kink near the active site, and the $\beta 4$ and $\beta 5$ sheets, Fig.6a). From the resulting analysis of the dynamic susceptibility of the various regions in the protein, it was concluded that the five selected regions showed increased flexibility in the substrate-free form which was a consequence of the fact that localized diffusion and jumps of atoms between 
minima (but excluding methyl rotations) are much more significant in substrate-free CYP101 than the camphor-bound form over the entire energy range investigated. Subsequent analysis of the MD simulation trajectories allowed identification of a network of key interactions comprising side-chain salt-bridges and hydrogen bonds that connect the five regions (Fig. 8). Weakening of these interactions was observed in the substrate-free form (relative to the camphor-bound form) consistent with increased conformational flexibility and increased interaction distances for several residues in these regions, which was also observed as inherent line-broadening of backbone amide resonances in the corresponding ${ }^{1} \mathrm{H}-{ }^{15} \mathrm{~N}$ HSQC NMR spectra for these residues. Interestingly, these five regions encompass the putative substrate access channel and it is possible that the dynamic modulation of the interaction network in these regions coupled to each other flexibility-wise provides the means to facilitate recognition and adaptation to diverse substrates, explaining the increased dynamic fluctuations manifested in the quasielastic neutron scattering spectra of substrate-free CYP101.

The success of the combined approach in addressing an important biological question of how protein dynamics modulates substrate binding in the CYP101 system augurs well for future application to other protein systems, including human P450s. Since the fast motions ultimately form a basis for slower motions that directly influence biological functions like substrate binding and enzymatic activity, future methodological improvements are likely to revolve around determining whether the fast motions are collective in nature and developing alternative combined approaches to investigate motions on the slower timescale. A possibility in this case would be to use newer NMR methods such as CPMG-based relaxation experiments in combination with neutron spin echo to obtain exchange rate constants that can then be physically interpreted via much longer MD simulations on the order of several microseconds. This would require preparation of large amounts of perdeuterated protein for such measurements, although this actually would be a benefit in that the same sample could be used for both neutron spin echo and NMR measurements, reducing the effort and cost for such experiments.
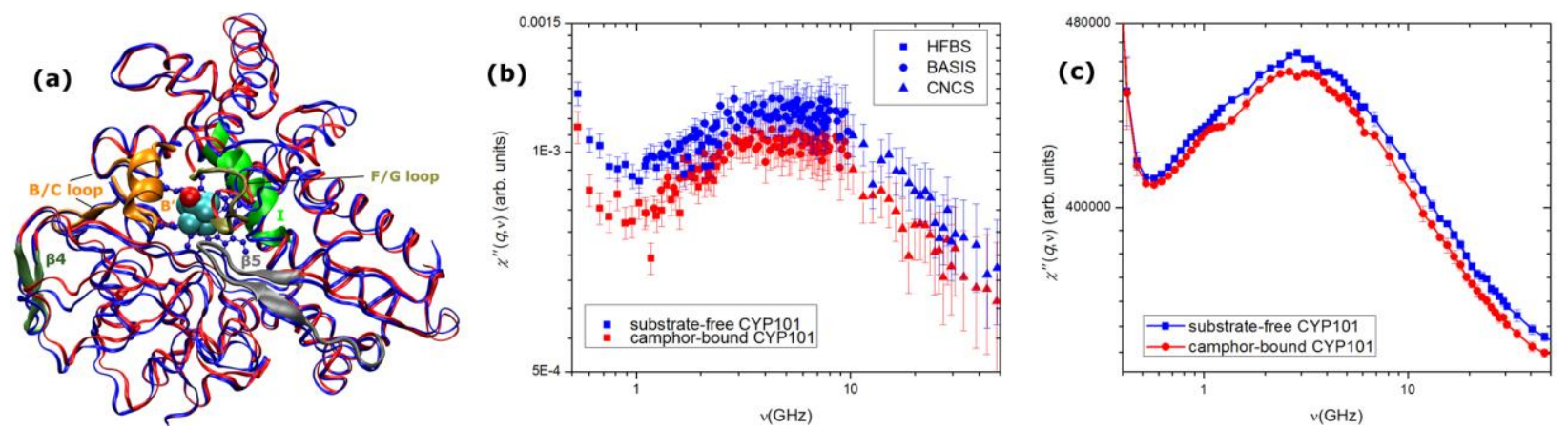

Figure 6 (a) X-ray crystal structure of camphor-bound (light-gray/red) CYP101, showing the putative substrate access channel lined by the five regions with the largest dynamic differences relative to the substrate-free (dark-gray/blue) form. (b) Dynamic susceptibility measurements for camphor-bound and substrate-free CYP101 obtained by combining by neutron scattering data from three instruments over a broad energy range - BASIS, HFBS and CNCS. (c) Dynamic susceptibility derived for camphor-bound and substrate-free CYP101 from MD simulations over the same energy range [62]. 
(a)
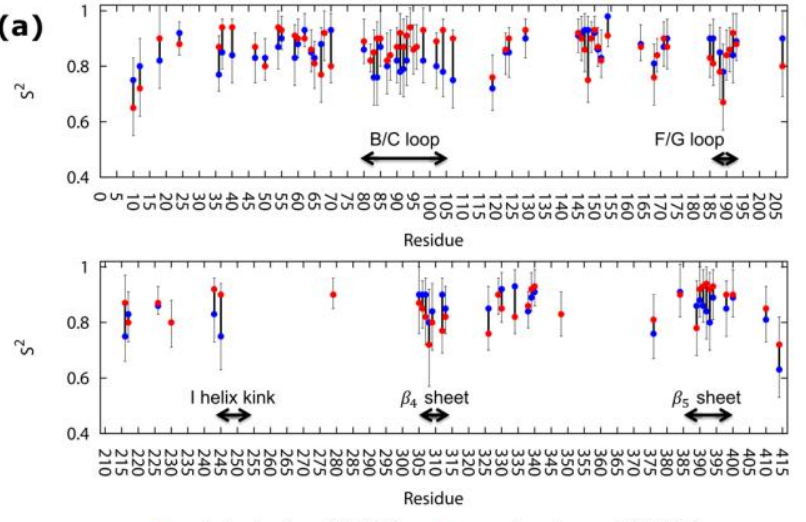

- substrate-free CYP101 • camphor-bound CYP101 (b)

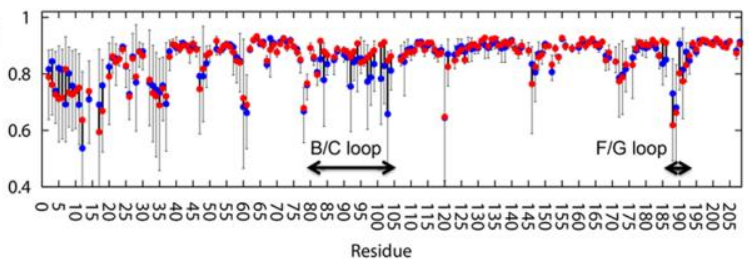

is

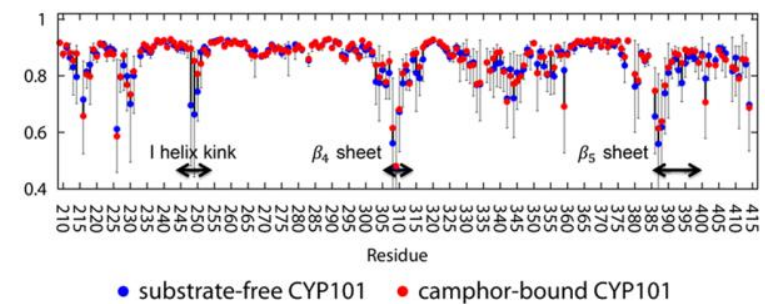

Figure 7 (a) Plot of NMR-derived order parameters versus amino acid sequence for substrate-free (darkgray/blue) and camphor-bound CYP101 (light-gray/red). Order parameters derived from MD simulations for the two protein forms are shown in $(b)$ for comparison [61].

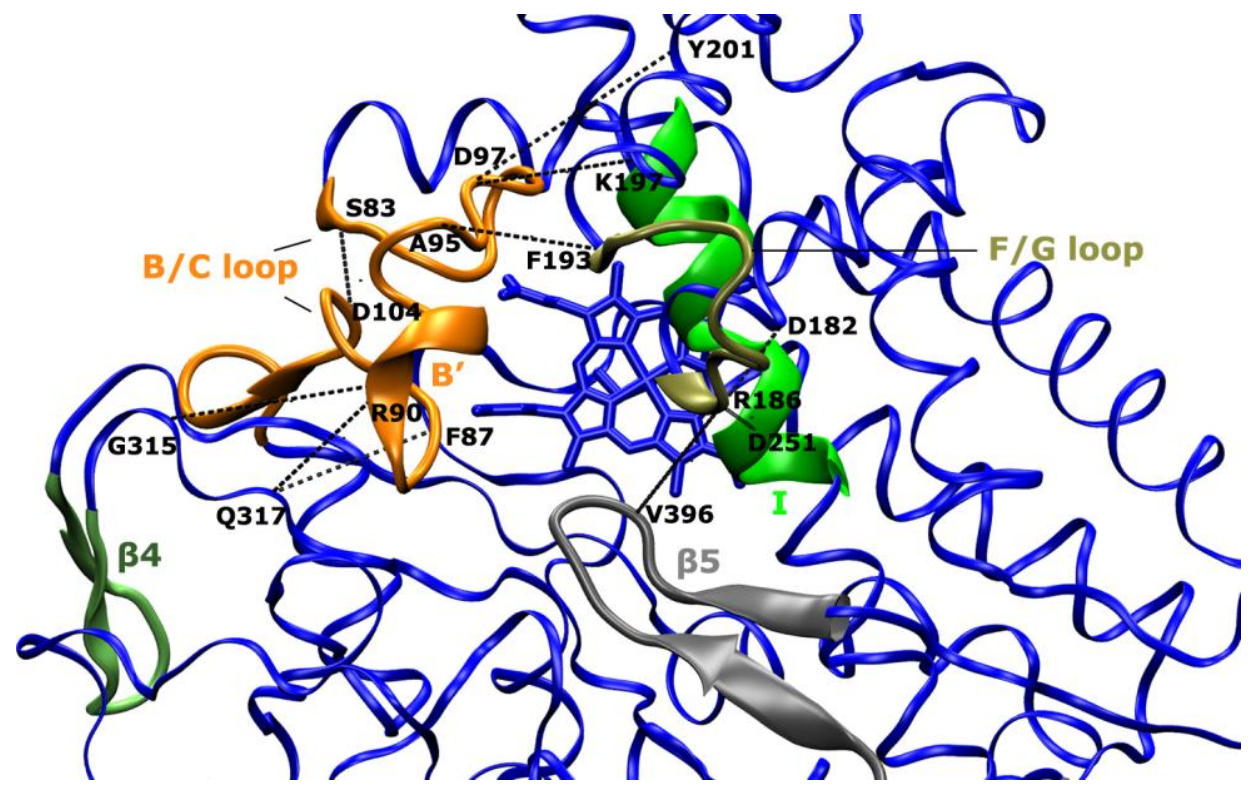

Figure 8 Network of key interactions connecting the various residues in the five selected regions that show the largest dynamic differences between camphor-bound and substrate-free CYP101 [61]. 


\section{Elastic Scattering and Mean-Square Displacements}

Our discussion so far has concerned the energy dependence of QENS i.e., the various quasielastic components that make up a typical energy spectrum. However, perhaps the most common use of QENS has been simply to determine the elastic scattering component, and from this the average mean-square displacement.

The average mean-square displacement is defined as

$$
<r^{2}>(t)=\frac{1}{N} \sum_{i=1}^{N}(r(t)-r(0))^{2}
$$

where $N$ is the number of atom and $t(\sim 1 /$ energy resolution $)$ is the approximate time limit of the instrument. In the experimental analysis, the commonly used expression $S(q, \omega=0) \sim I(q, t=\infty) \approx \exp \left(-q^{2}\left\langle r^{2}\right\rangle / 3\right)$ involves making the Gaussian approximation, which is valid for $q \rightarrow 0$. Based on this expression, elastic incoherent scattering can be used to determine $\left\langle\mathrm{r}^{2}(\mathrm{t})\right\rangle .\left\langle\mathrm{r}^{2}(\mathrm{t})\right\rangle$ is extracted by analyzing the elastic component of the incoherent dynamic structure factor as a function of $q$;

$$
<r^{2}>=-3 \frac{d \ln S(q, \omega=0)}{d q^{2}}
$$

The MSD averaged over the hydrogen atoms in a protein has been extensively investigated in this way $[7,67-70]$.

Some comment is merited on the temperature dependence of the MSD. At low temperatures, quasielastic scattering is of very low intensity, and correspondingly MSDs are small, originating primarily from inelastic vibrational scattering in proteins. Methyl group rotation is activated in proteins from $\sim 100 \mathrm{~K}$ upwards and is hydration-independent $[8,12,14]$. At higher temperatures $(180 \sim 220 \mathrm{~K})$ a further, hydration-dependent transition occurs [8,10,12-14], during which there is simulation evidence that jumps between minima are activated [71].

The temperature dependence of the nanosecond dynamics of different chemical classes of amino-acid residue has been analyzed for cytochrome P450cam [72]. The $\left\langle\mathrm{r}^{2}(\mathrm{t})\right\rangle$ of the nonexchangeable hydrogen atoms in the protein calculated from MD simulations at $1 \mathrm{~ns}$ was found to agree quantitatively with the experimental data. The MSD exhibits one transition at $T \sim 150 \mathrm{~K}$ and another in only the hydrated sample at $T \sim 180-220 \mathrm{~K}$.

The contributions of different residue types in the protein to the two dynamic transitions are of particular interest. As shown in Fig. 9, at T 100-160 K anharmonic motion in hydrophobic and aromatic residues is activated whereas, due to hydrogen-bonding interactions, hydrophilic residue motions are suppressed. In contrast, at $T \sim 180-220 \mathrm{~K}$ water-activated jumps of hydrophilic side chains, which are strongly coupled to the relaxation rates of the hydrogen bonds they form with hydration water, become apparent. Thus, with increasing temperature, first the hydrophobic core awakens, followed by the hydrophilic surface [72]. 

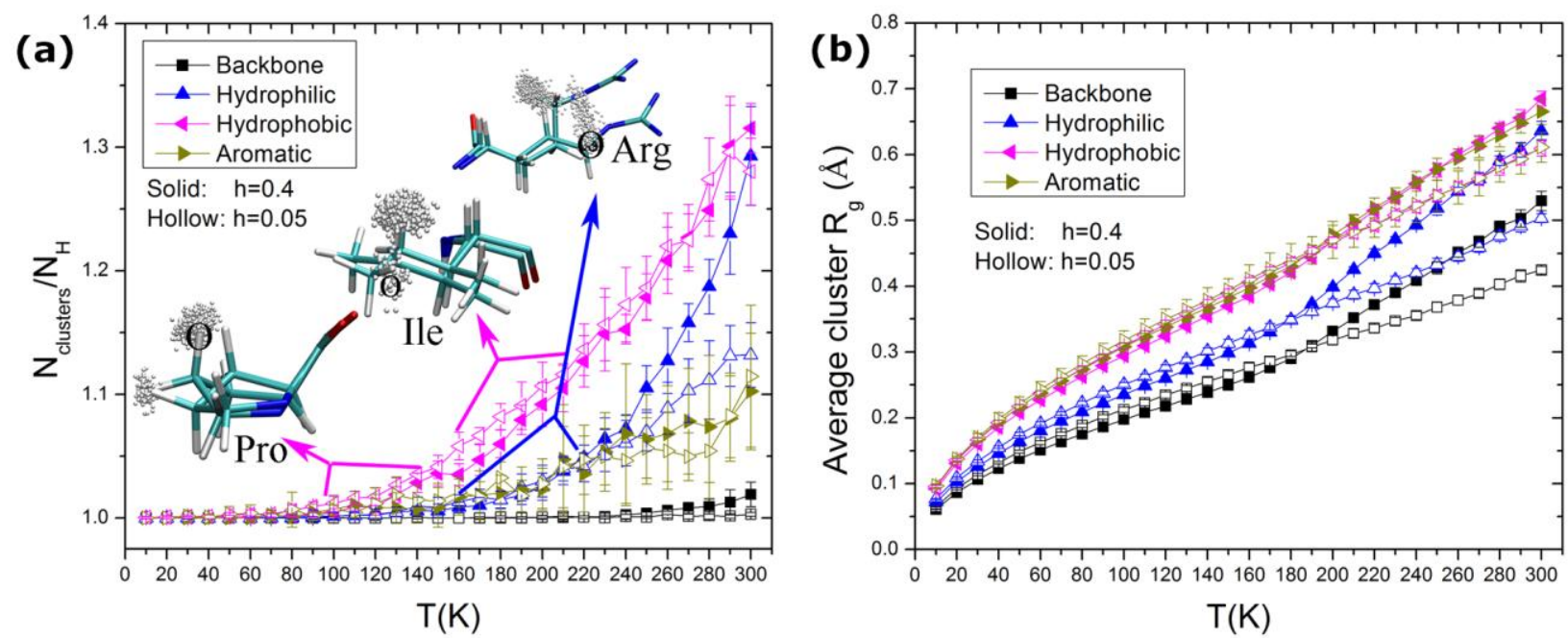

Figure 9 Temperature dependence of (a) average number of clusters per hydrogen atom and (b) the average cluster radius of gyration, calculated for non-exchangeable hydrogen atoms in different types of residue. The clusters were obtained from scatter plots of individual hydrogen atom trajectories. Insets show example jumps of non-exchangeable hydrogen atoms in Pro, Ile and Arg side chains as labeled [72].

\section{Protein Dynamics at Very Long Times}

The MSD extracted from the elastic component of the QENS DSF, S(q, $\omega=0)$, depends on the energy resolution of the instrument used [73-75]. Instruments with low energy resolution will detect only fast motions. In contrast, the 'intrinsic' MSD is the long-time, converged value, defined as the MSD that appears in the infinite time limit of $I(q, t)$ in Eq. (1). This corresponds to the value that would be obtained at infinite energy resolution. In Ref. [76], a method was developed to extract an approximate value for the intrinsic, long-time, $t \rightarrow \infty$, value of the MSD from resolution-dependent neutron scattering data. This method involves fitting a model of the incoherent elastic resolution broadened DSF, $S_{R}(q, \omega=0)$,

$$
S_{R}(q, \omega=0)=I(q, t=\infty)+(1-I(q, t=\infty)) \frac{W}{W+\lambda}+A
$$

to the normalized elastic incoherent DSF data, where $I(q, t=\infty)=\exp \left(-\left\langle r^{2}>q^{2} / 3\right)\right.$ is the Debye Waller factor, $W$ is the energy resolution width of the neutron scattering instrument, $\lambda(\sim 1 / \tau)$ is the relaxation frequency and $A$ is a constant. In the model in Eq. (8), it is assumed that cumulants beyond Gaussian term are negligible and all atoms have the same MSD i.e., neglecting the dynamical heterogeneity. Using this model, the intrinsic long time value of MSD is determined from the resolution dependent data. The intrinsic MSD is independent of the energy resolution width.

Eq. (8) was used to obtain the intrinsic $\left\langle\mathrm{r}^{2}\right\rangle$ of heparin sulphate (HS), ribonuclease A (RNA) and staphylococcal nuclease (SNase) from published neutron scattering data [76]. The application of the method in Ref. [76] was expanded to obtain the long-time $\left\langle r^{2}\right\rangle$ from finitetime MD simulations [77]. In this method, $I(q, t)$ is represented as 


$$
I_{m}(q, t)=I(q, t=\infty)+(1-I(q, t=\infty)) C(t)
$$

and this function is fitted to the simulation-derived $I(q, t)$ to obtain the intrinsic $\left\langle\mathrm{r}^{2}\right\rangle$, where $I(q, t=\infty)=\exp \left(-\left\langle r^{2}\right\rangle q^{2} / 3\right)$ is the time-independent part, $(1-I(q, t=\infty)) C(t)$ is a time-dependent part, and the correlation function $C(t)=\exp \left(-(\lambda t)^{\beta}\right.$ is a stretched exponential function. A stretched exponential function is equivalent to a superposition of exponential decay functions representing individual diffusive processes. The model in Eq. (9) contains the intrinsic long-time MSD, the decay-rate parameter $(\lambda)$ and the stretched exponential parameter $(\beta)$.
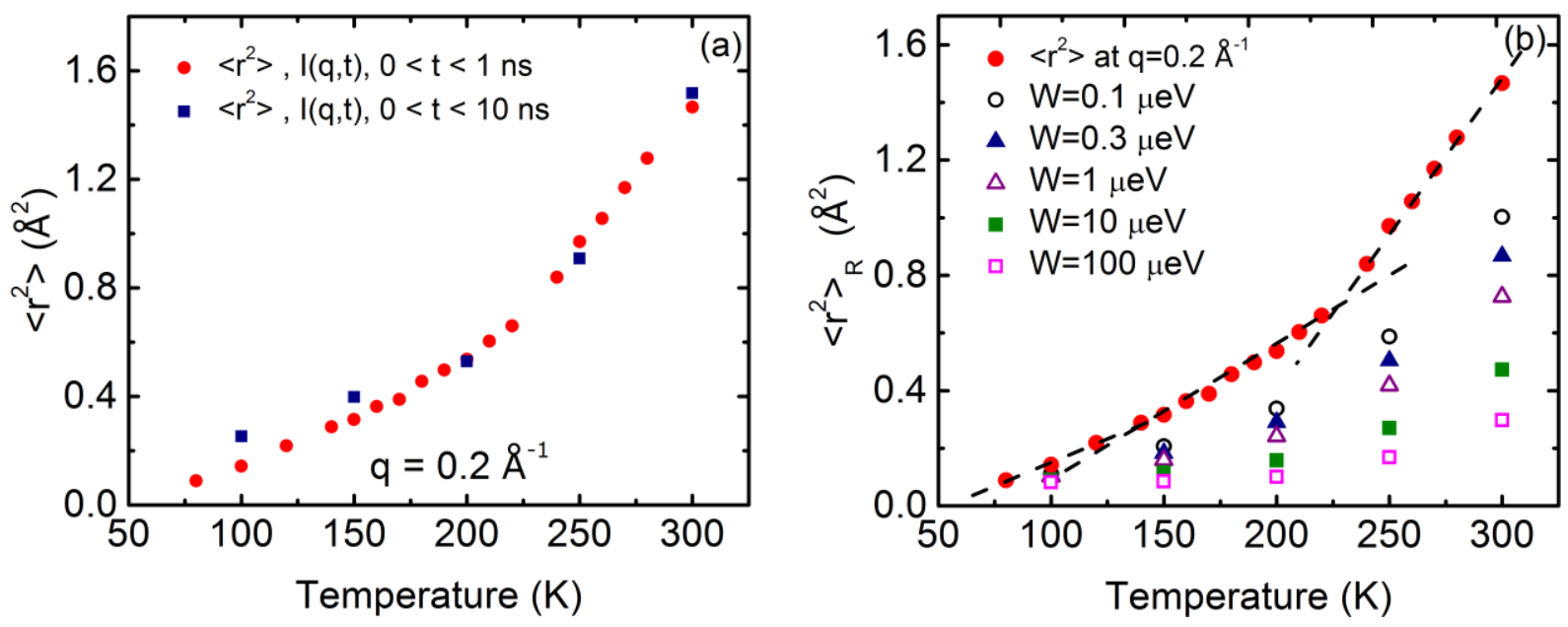

Figure 10 (a) The long time MSD versus temperature obtained from the fits to the $I(q, t)$ obtained from 100ns (solid circles) and $1 \mu$ s (solid squares) simulations. (b) Comparison of the long time MSD and the resolution broadened MSD calculated from the model in Eq. (9) for the energy resolution widths, $W=$ $0.1,0.3,1,10$, and $100 \mu \mathrm{eV}$ [77].

This method is illustrated for hydrated lysozyme by performing two simulations of different lengths, $\mathrm{t}=100 \mathrm{~ns}$ and $1 \mu \mathrm{s}$, and then, using the SASSENA software [27] to, directly calculating the $I(q, t)$ up to 1 and $10 \mathrm{~ns}$, respectively. The model $I_{m}(q, t)$ was then fitted to the simulation-derived $I(q, t)$. The resulting $\left\langle\mathrm{r}^{2}\right\rangle$ was found to be independent of the simulation time used to calculate $I(q, t)$, at least up to $1 \mu \mathrm{s}$ (Fig. 10a), consistent with $\left\langle\mathrm{r}^{2}\right\rangle$ representing a timeindependent, long-time MSD. The resolution broadened MSD, $\left\langle\mathrm{r}^{2}\right\rangle_{\mathrm{R}}$ was also calculated from the resolution broadened DSF $S_{R}(q, \omega)$ with different energy resolution widths $\left(\mathrm{W} \sim 1 / \tau_{\mathrm{R}}\right)$. The intrinsic $\left\langle\mathrm{r}^{2}\right\rangle$ was found to be approximately twice $\left\langle\mathrm{r}^{2}\right\rangle_{\mathrm{R}}$, the MSD calculated for motions up to $1.5 \mathrm{~ns}(\mathrm{~W}=1 \mu \mathrm{eV})$ (Fig. 10b).

According to the modeling implicit in Fig. 10, the existence of the dynamical transitions is not an artificial consequence of finite instrumental resolution $W$ and a correspondingly limited time window. However, the instrumental energy resolution does have a significant effect on the theoretical $\mathrm{T}_{\mathrm{D}}$, shifting it to higher temperature with increasing energy resolution width [73$75,78,79]$. The impact of the energy resolution width (W) on the MSD is determined by the decay rate of the correlations, which depends on the relaxation frequency parameter $\lambda(1 / \tau)$ in the stretched exponential function $\mathrm{C}(\mathrm{t})$. 
One interesting feature of neutron scattering experiments is that $S(q, \omega)$ depends on $q$, and thus furnishes information on the geometry of motions. Moreover, the average MSD extracted from measured neutron scattering data has been found to be $q$-dependent [80], and $q$-dependence was also found in the intrinsic MSD [77]. A $q$-dependent MSD could in principle arise from (i) neglecting the higher cumulant terms in the complete expression for $I(q, t)$ in Eq. (1) and retention of only the Gaussian term in the model of $I(q, t)$ : includes all cumulant terms, whereas the $I_{m}(q, t)$ model in Eq. (9) includes only the Gaussian term; and/or (ii) neglecting the heterogeneity of hydrogen atom motions in the protein: the $I(q, t)$ in Eq. (1) is calculated as sum over many hydrogens, but the scattering from different hydrogens is represented by a single scatterer in the $I_{m}(q, t)$ model in Eq. (9) .

In Ref. [81], the origin of the $q$-dependence of the extracted MSD was investigated. The impact of the Gaussian approximation was tested by calculating the full $I(q, t)$ in Eq.(1) and by employing the Gaussian approximation :

$$
I_{G}(q, t)=\frac{1}{Z} \sum_{j}^{Z} b_{j, \text { inc }}{ }^{2} \exp \left(-\frac{1}{2}\left\langle\left\{q \cdot\left[r_{j}(t)-r_{j}(0)\right]\right\}^{2}\right\rangle\right) .
$$

The model in Eq. (9) was applied to both $I(q, t)$ in Eq.(1) and $\mathrm{I}_{\mathrm{G}}(\mathrm{q}, \mathrm{t})$, in Eq. (10) for all hydrogen atoms from a simulation of lysozyme. It was found that the difference between the MSD obtained from the $I(q, t)$ and $I_{G}(q, t)$ is insignificant, and the MSD is still $q$-dependent in both cases. This analysis thus shows that the q-dependence of MSD does not arise from the use of the Gaussian approximation.

The second possible origin was investigated by fitting the $I_{m}(q, t)$ model in Eq. (9) to the simulation-derived $I(q, t)$ for individual hydrogen atoms in lysozyme. The analysis of the simulation-derived $I(q, t)$ leads to q-independent values of the MSD for these individual hydrogen atoms, indicating that the $q$-dependence arises from neglecting the dynamical heterogeneity.

An obstacle in interpreting $I(q, t)$ for QENS lies in the fact that the overall total signal contains not only the internal motions, but also global translational and rotational dynamics of the whole macromolecule. In the $q$ range studied by neutron spin echo $\left(0.1\right.$ to $\left.2 \mathrm{~nm}^{-1}\right)$, the global translation and rotation of the whole protein molecule dominates the dynamic signal measured. The QENS study on domain fluctuations in proteins by Ref. [47] showed that, for small $q$ values such that $1 / q$ is larger compared to the overall protein dimension, $I(q, t)$ decays as a single exponential, consistent with the fact that the dominant motion on this length scale is global (Brownian) diffusion of the whole protein molecule in solution. However, once $q$ values become larger, such that $1 / q$ is smaller than the overall protein dimension, the signal becomes dominated by the internal motion of the protein and, $I(q, t)$ starts to deviate from single exponential behavior towards a longer-tailed relaxation, indicating the presence of more complex relaxation behavior.

\section{Nonequilibrium considerations}

In the practical interpretation of $I(q, t)$, Brownian dynamics is often assumed for the internal protein motion [47,49]. This assumption implies that protein dynamics is at thermodynamic equilibrium and ergodic, and, therefore, that the ensemble averaged dynamics, such as relaxation time values, etc. extracted from the scattering function reflects the average 
dynamical behavior of the individual protein molecules. This is a valid assumption for a large collection of molecules, but the question arises as to its applicability at the single-molecule level.

(a)

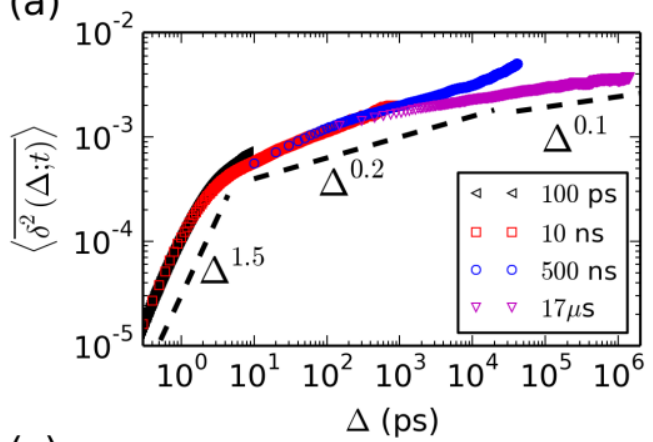

(c)

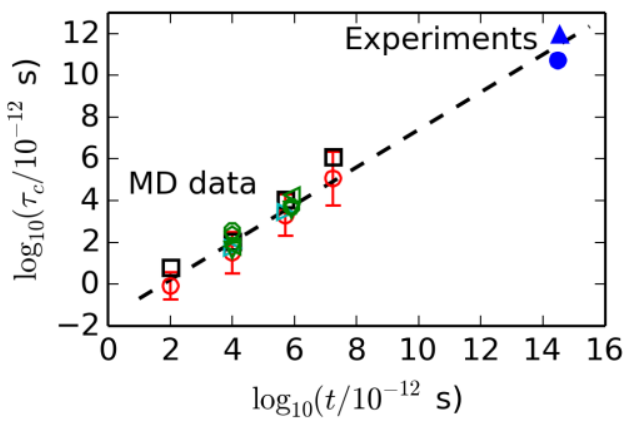

\begin{tabular}{|c|c|}
\hline$\square$ ㅁ PGK domains & ○ ePepN, dom. 2-4 \\
\hline O $\bigcirc$ PGK residues & O $\mathrm{O}$ ePepN, dom. 4-rest \\
\hline$\triangleright \triangleright$ K-RAS segments & Ref. 86 \\
\hline$\triangleleft \triangleleft$ ePepN, dom. 1-2 & $\boldsymbol{\Delta} \boldsymbol{\Delta}$ Ref. 83 \\
\hline$\nabla \nabla$ ePepN, dom. 2-3 & - $\cdot \propto t^{0.9}$ \\
\hline
\end{tabular}

(b)

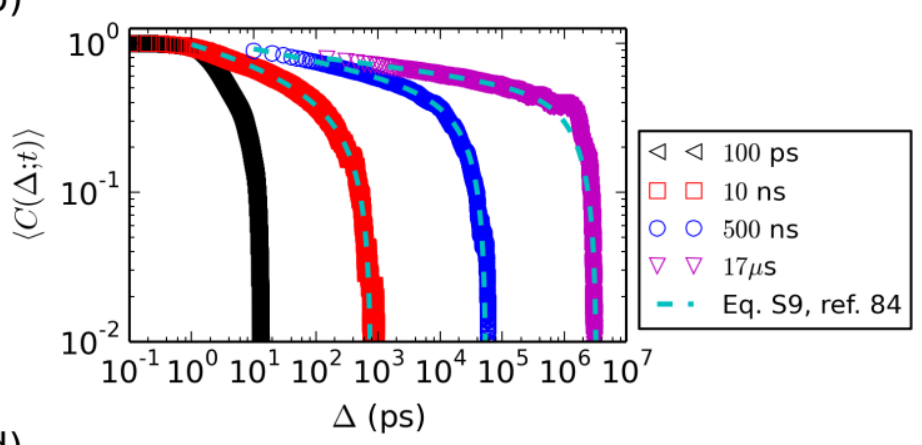

(d)

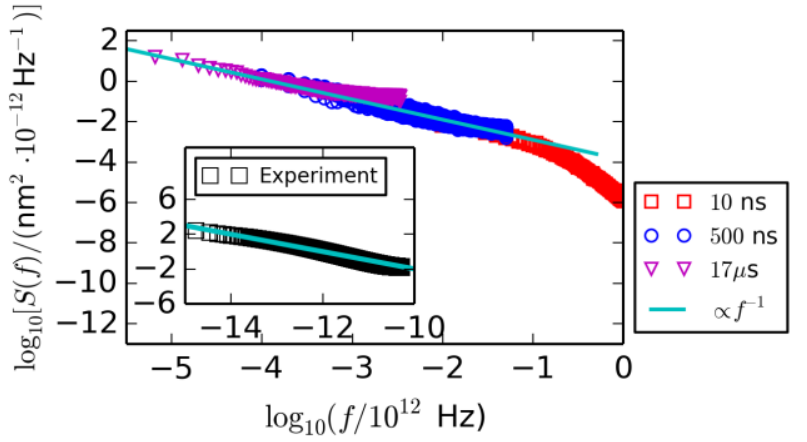

Figure 11 MD simulation data of internal inter-domain dynamics of the enzyme PGK. (a) The time averaged MSD of the inter-domain motion, defined by center-of-mass distance fluctuation between the domains, measured at different observation time length. (b) The normalized autocorrelation function of the inter-domain motion obtained at different observation times. (c) The logarithm of the characteristic time of the motion plotted against the logarithm of the observation time. (d) The power spectral density of the inter-domain motion in log-log scale. See Ref. [84] for details.

In the light of single-molecule experimental data [82,83] and recent MD results [84], the Brownian assumption for the single protein internal dynamics becomes questionable. In Ref. [84] a detailed MD simulation study showed that large scale internal dynamics in single globular protein molecules, such as inter-domain motions, are self-similar and non-ergodic. Despite the confinement imposed by the overall average protein structure, the time-averaged mean square displacement (TA-MSD) of the inter-domain distance fluctuation does not reach a stationary value but increases according to a power-law with an exponent of roughly 0.1 , even on the micro-second time scale (Fig. 11). The relaxation function of the corresponding dynamics was found to be consistent with the fractional Fokker-Planck equation, describing a non-ergodic diffusion process in an external potential [85]. The power spectrum of the corresponding motions exhibits a $1 / \mathrm{f}$ noise behavior for timescales beyond $p s$, spanning over at least 5 decades in time. Furthermore, broken ergodicity is observed not only in the global, inter-domain fluctuations, but also in the local dynamics indicated by the inter-residue distance fluctuations. As a consequence 
of the ergodicity breaking, protein dynamics exhibits aging, i.e., the relaxation function measured at a given observation time window shifts towards a longer time range if the observation time becomes longer, and the characteristic time of the motion, $\tau_{\mathrm{c}}$, depends on the length of the observation time $t$, increasing in a power-law fashion as $\tau_{\mathrm{c}} \sim t^{0.9}$.

The notion of non-ergodic internal protein dynamics have significant impact on the interpretation of the data obtained from neutron scattering. In the presence of ergodicity breaking, ensemble and time averages are no longer the same. Thus, the dynamical behavior of individual protein molecules can no longer be completely represented by ensemble averaged observations, such as $I(q, t)$. Furthermore, the strict Brownian assumption for the internal dynamics is mostly likely inaccurate, and novel theoretical models for interpreting the overall scattering signal, incorporating non-Brownian internal motion are needed extending to timescales comparable to the physiological lifespan of globular proteins in cells.

\section{Collective membrane dynamics probed by neutron spin echo (NSE)}

Finally, we examine the application of QENS to large biological systems, namely biological membranes. Motions in biomembranes also occur on a wide variety of length and time scales, including fast local fluctuations in the picosecond and Angstrom ranges to bilayer undulation and bending dynamics, occurring on nanosecond time scales and tens of nanometer length scales. Long wavelength undulation motions in lipid bilayers have been investigated using NSE spectrometers.

Typically, the $I(q, t)$ data measured with NSE are analyzed using a modified approach introduced by Zilman and Granek [87]. Briefly, the normalized ISF is fitted in the time domain using a stretched exponential decay,

$$
\frac{I(q, t)}{I(q, 0)}=A e^{-(\Gamma(q) \cdot t)^{2 / 3}}
$$

where $A$ is a normalization constant and $\Gamma(q)$ is the relaxation rate, which can be related to the bilayer bending modulus $K_{c}$ through the following relationship.

$$
\Gamma(q)=0.0058\left(\frac{k_{B} T}{K_{c}}\right)^{\frac{1}{2}} \frac{k_{B} T}{\eta} q^{3}
$$

where $\eta$ is the solvent viscosity. It is evident from Eq. (12) that a plot of $\Gamma(q) / q^{3}$ is q independent, which is inversely proportional to the square root of $K_{c}$.

Recently, NSE has been combined with neutron scattering length density (NSLD) contrast matching to unambiguously determine the mechanical properties of the nanoscopic lipid heterogeneities populating unilamellar lipid vesicles [88]. Certain lipid mixtures exhibit liquidliquid phase coexistence forming two phases: the liquid ordered $\left(\mathrm{L}_{\mathrm{O}}\right)$ phase, which is enriched in high melting lipids and cholesterol and thought to be similar to the composition of lipid rafts in mammalian cells, and the liquid disordered $\left(\mathrm{L}_{\mathrm{D}}\right)$ phase that is thought to surrounds rafts. The model systems were composed of cholesterol (Chol), 1,2-distearoyl-sn-glycero-3phosphocholine (DSPC) and 1-palmitoyl-2-oleoyl-sn-glycero-3-phosphocholine (POPC). The 
compositions of the coexisting $\mathrm{L}_{\mathrm{D}}$ and $\mathrm{L}_{\mathrm{O}}$ phases were obtained from the ternary phase diagram, and their relative abundance in the nano-domain containing sample was obtained from the tie line. Specifically, domains of radius 5-7 nm are formed in a mixture of Chol, DSPC and POPC in a 22:39:39 ratio. In the nano-domain containing sample, based on its difference in composition ratios, the NSLD of each phase was tuned through judicious hydrogen/deuterium substitution of the constituent lipids such that the NSLD of one phase could be matched to that of the solvent by also varying the $\mathrm{D}_{2} \mathrm{O} / \mathrm{H}_{2} \mathrm{O}$ ratio of the solvent. This has enabled neutron techniques to isolate the scattering from only the non-contrast matched phase.

NSE measurements were performed on four samples at $20{ }^{\circ} \mathrm{C}$ : $\mathrm{L}_{\mathrm{O}}$ only vesicles in $\mathrm{D}_{2} \mathrm{O}$ $\left(\mathrm{L}_{\mathrm{O}}\right), \mathrm{L}_{\mathrm{D}}$ only vesicles in $\mathrm{D}_{2} \mathrm{O}\left(\mathrm{L}_{\mathrm{D}}\right)$, coexisting $\mathrm{L}_{\mathrm{O}} / \mathrm{L}_{\mathrm{D}}$ vesicles with no contrast enhancement in $\mathrm{D}_{2} \mathrm{O}$ (Domains), and $\mathrm{L}_{\mathrm{D}}$ nano-domains in 93.5\% $\mathrm{D}_{2} \mathrm{O} / \mathrm{H}_{2} \mathrm{O}$ (Domains- $\mathrm{L}_{\mathrm{D}}$ ). The measured relaxation rates $\Gamma(q)$ did show a $q^{3}$ dependence in the $q$-range of $0.05-0.11 \AA^{-1}$ for all four samples. Not surprisingly, the bending moduli derived from the data further showed that the $\mathrm{L}_{\mathrm{O}}$ sample is approximately one order of magnitude more rigid than the $\mathrm{L}_{\mathrm{D}}$ sample. Furthermore, the NSE experiments revealed that the modulus of the nano-domains (Domains- $\mathrm{L}_{\mathrm{D}}$ ) is indistinguishable from that of the pure (single phase) $\mathrm{L}_{\mathrm{D}}$ sample, while the bending modulus of the non-contrast matched domains sample (Domains) has an intermediate rigidity, simply reflecting the ensemble nature of the measurement.

Although quasi- and in-elastic neutron scattering gives wave vector resolved access to membrane molecular dynamics, as discussed above for proteins, it remains a challenge to be able to unambiguously assign a scattering signal to a particular dynamical mode. To overcome this, MD simulations were again used to decompose the individual molecular motions observed in lipid bilayer systems, and thus help assign the measured neutron signals to certain motions of molecules or molecular components. All-atom MD simulations were performed on three symmetric planar bilayer patches, compositionally similar to those studied experimentally, namely: $\mathrm{L}_{\mathrm{O}}, \mathrm{L}_{\mathrm{D}}$, and $\mathrm{L}_{\mathrm{D}}$ nano-domains coexisting in an $\mathrm{L}_{\mathrm{O}}$ bilayer. In order to study nano-domain properties, an in-register, $6.8 \mathrm{~nm}$ radius $\mathrm{L}_{\mathrm{D}}$ domain surrounded by an $\mathrm{L}_{\mathrm{O}}$ phase was assembled.

With MD simulation trajectories, the individual contributions to the total observed dynamics of various motions, such as the thickness, undulational and lateral fluctuations, can be easily decomposed as

$$
\frac{I(q, t)}{I(q, 0)}=\left(\frac{I(q, t)}{I(q, 0)}\right)_{t f} \times\left(\frac{I(q, t)}{I(q, 0)}\right)_{\text {und }} \times\left(\frac{I(q, t)}{I(q, 0)}\right)_{l a t}
$$

where $I(q, t) / I(q, 0)_{\mathrm{tf}}$ is the ISF from thickness fluctuations, $I(q, t) / I(q, 0)_{\text {und }}$ describes bilayer undulational dynamics and $I(q, t) / I(q, 0)_{\text {lat }}$ accounts for in-plane fluctuations. The corresponding ISFs were computed by reducing the simulated bilayer to pseudo-atoms spread from the head group areas of the two leaflets (the bilayer thickness) and the center of mass of the bilayer (the bilayer undulation) in $z$. The pseudo-atom MD trajectories were locally averaged and calculated on an $\mathrm{M} \times \mathrm{M}$ grid (roughly $10 \AA$ between grid points). The mapping procedure was described in [88], which was adapted from Ref. [89].

The decomposition of MD trajectories showed that the thickness fluctuations of the bilayer occur with a characteristic frequency which contributes to the ISF at $q$ values corresponding to the bilayer thickness, whereas the lateral fluctuations occurs in much larger $q$ 
window and are thus not expected to contribute to the ISF of relevance here. Therefore, the corrected undulational dynamics was obtained by removing the contribution of bilayer thickness fluctuations from the total all-atom ISF according to

$$
\left(\frac{I(q, t)}{I(q, 0)}\right)_{\text {und }}=\left(\frac{I(q, t)}{I(q, 0)}\right) /\left(\frac{I(q, t)}{I(q, 0)}\right)_{t f}=A_{\text {und }} e^{-\left(\Gamma_{\text {und }}(q) \cdot t\right)^{2 / 3}}
$$

Overall, the trend in the undulational dynamics obtained from MD simulations (Fig. 12) is qualitatively consistent with that obtained in the NSE experiments (Fig. 4A-D in ref. [88]). However, it should be noted that due to limitations imposed by the simulation box size and duration, the long wavelength undulation modes observed experimentally cannot captured by the MD, which therefore yields only a qualitative comparison. Nevertheless, the simulation results confirmed the experimental findings that the pure $\mathrm{L}_{\mathrm{O}}$ patch is less dynamic than the pure $\mathrm{L}_{\mathrm{D}}$ patch, and the nano-domain region is more dynamic than the average of the full domain containing patch (Fig. 13). The combined NSE experiments and MD simulations revealed a dynamical decoupling of the nanodomains from the surrounding phase, suggesting that mechanical properties connected to the local composition persist even at the nanometer length scale.
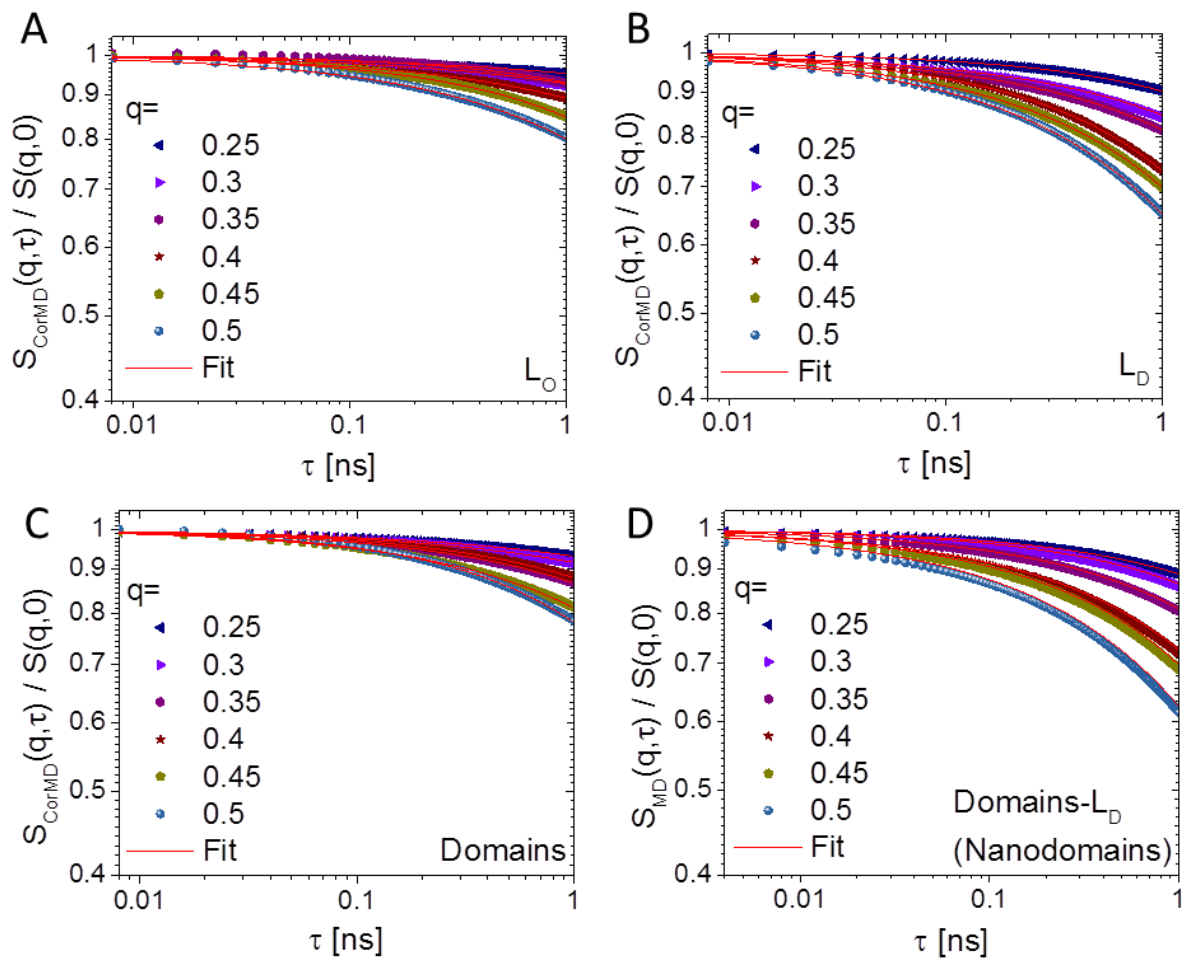

Figure 12 Undulational ISFs calculated from MD simulations of (A) the $\mathrm{L}_{\mathrm{O}}$ patch, (B) the $\mathrm{L}_{\mathrm{D}}$ patch, (C) the full domain containing patch, and (D) the $\mathrm{L}_{\mathrm{D}}$ nano-domain only in the domain containing patch (Fig. $\mathrm{S} 2$ in ref. [88]). The ISF was computed from the MD trajectories using the SASSENA [27] from 0.08 ps to $1 \mathrm{~ns}$, and from 0.25 to $0.5 \AA^{-1}$, a range based upon the constraints imposed by the simulation box dimensions and sampling timescales with sufficient statistics. 

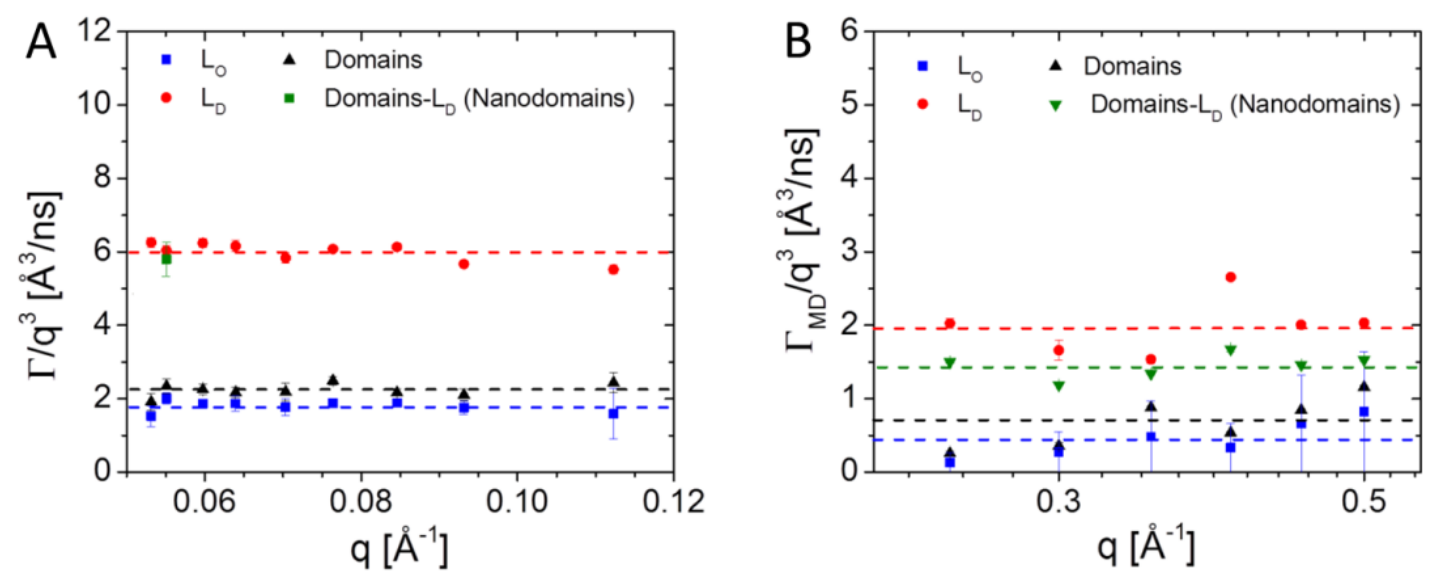

Figure 13 Fitted undulational relaxation times as a function of $q$ obtained from experiments (A) and allatom MD simulations (B) [88].

\section{Conclusions and Future Perspectives}

A recurring theme throughout this review has been the need to insert molecular dynamics simulation as a stepping stone between QENS experiment and any attempt at a simple description of the dynamics of biomaterials. When this is done, an unambiguous interpretation of QENS spectra can be obtained. For proteins, this interpretation is often usefully made in terms of an energy landscape picture, and the complexity of this means that elements of complex systems theory, such as network analysis, will likely be increasingly employed in the future.

QENS sits at several cross-roads. Firstly, there is the intersection of physics and biology. Concepts from soft matter physics (such as the glass transition [21]) will filter through to biology. However, biologically-driven questions should be asked. In this context it is interesting to note the recent proposal of a database of neutron data from biological neutron experiments, which would be useful to reduce replication (saving beam time) and to have more data easily available for analysis [90]. Finally, QENS connects atomic-level behavior to collective motions and is thus part of a broad multiscale puzzle. The recent paper on nonequilibrium aspects of protein dynamics [84] illustrates how concepts derived from local, atomic-scale motions can sometimes be usefully generalized over many orders of magnitude. Connecting scales is the great challenge for the future for biological physics.

\section{$\underline{\text { Acknowledgement }}$}

This research is funded by the Genomic Science Program, Office of Biological and Environmental Research, U. S. Department of Energy (DOE). This research used resources of the Oak Ridge Leadership Computing Facility at the Oak Ridge National Laboratory, which is supported by the Oce of Science of DOE under Contract No. DE-AC05-00OR22725. 


\section{References}

[1] H. Frauenfelder, S.G. Sligar, and P. G. Wolynes, The energy landscapes and motions of proteins, Science 254, 1598 (1991).

[2] C. D. Snow, H. Nguyen, V. S. Pande, and M. Gruebele, Absolute comparison of simulated experimental protein-folding dynamics. Nature (London) 420, 102 (2002).

[3] R. Biehl, B. Hoffmann, M. Monkenbusch, P. Falus, S. Préost, R. Merkel, and D. Richter, Direct Observation of Correlated Interdomain Motion in Alcohol Dehydrogenase, Phys. Rev. Lett. 101, 138102 (2008).

[4] L. Zhu, D. Frenkel, and P. G. Bolhuis, Role of Fluctuations in Ligand Binding Cooperativity of Membrane Receptors, Phys. Rev. Lett. 106, 168103 (2011).

[5] J. Pieper, A. Buchsteiner, N. A. Dencher, R. E. Lechner, and T. Hauß, Transient Protein Softening during the Working Cycle of a Molecular Machine, Phys. Rev. Lett. 100, 228103 (2008).

[6] F. Piazza, P. De Los Rios, and F. Cecconi, Temperature Dependence of Normal Mode Reconstructions of Protein Dynamics, Phys. Rev. Lett. 102, 218104 (2009).

[7] W. Doster, S. Cusack, and W. Petry, Dynamic instability of liquidlike motions in a globular protein observed by inelastic neutron scattering, Phys. Rev. Lett. 65, 1080 (1990).

[8] J. H. Roh, J. E. Curtis, S. Azzam, V. N. Novikov, I. Peral, Z. Chowdhuri, R. B. Gregory, and A. P. Sokolov, Influence of Hydration on the Dynamics of Lysozyme, Biophys. J. 91, 2573 (2006).

[9] D. E. Shaw, Millisecond-Scale Molecular Dynamics Simulations on Anton, Proceedings of the ACM/IEEE Conference on Supercomputing (SC09), Portland, Oregon, 2009.

[10] G. Zaccai, How Soft Is a Protein? A Protein Dynamics Force Constant Measured by Neutron Scattering, Science 288, 1604 (2000).

[11] J. Fitter, R. E. Lechner, G. Buldt, and N. A. Dencher, Internal molecular motions of bacteriorhodopsin: hydration-induced flexibility studied by quasielastic incoherent neutron scattering using oriented purple membranes, Proc. Natl. Acad. Sci. U.S.A. 93, 7600 (1996).

[12] J. H. Roh, V. N. Novikov, R.B Gregory, J. E. Curtis, Z. Chowdhuri, and A. P. Sokolov, Onsets of Anharmonicity in Protein Dynamics, Phys. Rev. Lett. 95, 038101 (2005).

[13] S. H. Chen, L. Liu, E. Fratini, P. Baglioni, A. Faraone, and E. Mamontov, Observation of fragile-tostrong dynamic crossover in protein hydration water, Proc. Natl. Acad. Sci. U.S.A. 103, 9012 (2006).

[14] W. Doster and M. Settles, Protein-water displacement distributions, Biochim. Biophys. Acta, Proteins Proteomics, Biochim. Biophys. Acta, Proteins Proteomics 1749, 173 (2005).

[15] S. Dellerue and M.-C. Bellissent-Funel, Relaxation dynamics of water molecules at protein surface, Chem. Phys. 258, 315 (2000).

[16] S. Khodadadi, S. Pawlus, J. H. Roh, V. Garcia Sakai, E. Mamontov, and A. P. Sokolov, The origin of the dynamic transition in proteins, J. Chem. Phys. 128, 195106 (2008). 
[17] G. R. Kneller, W. Doster, M. Settles, S. Cusack, and J. C. Smith. Methyl group dynamics in the crystalline alanine dipeptide: A combined computer simulation and inelastic neutron scattering analysis, J. Chem. Phys. 97(12), 8864-8879 (1992).

[18] V. Recceveur, P. Calmettes, J. C. Smith, M. Desmadril, G. Coddens, and D. Durand, Picosecond dynamical changes on denaturation of yeast phosphoglycerate kinase revealed by quasielastic neutron scattering, Proteins: Structure,Functions, and Genetics 28, 380-387 (1997).

[19] J. C. Smith, Protein dynamics: comparison of simulations with inelastic neutron scattering experiments, Quart. Rev. Biophys. 24, 227-291 (1991).

[20] J. C. Smith, Inelastic and quasielastic neutron scattering: complementarity with biomolecular simulation, Structure and Dyanmics of Bionolecules: Neutron Synchroton Radiation for Condensed Matter Studies, Oxford University Press, Oxford, UK 161-180 (2000).

[21] S. Khodadadi and P. Sokolov, Protein dynamics: from rattling in a cage to structural relaxation, Soft Matter 11, 4984-4998 (2015).

[22] T. Róg, K. Murzyn, K. Hinsen, G.R. Kneller, nMoldyn: a program package for a neutron scattering oriented analysis of molecular dynamics simulations, J. Comp. Chem. 24(5), 657-667 (2003).

[23] A. Micu, and J. C. Smith, SERENA: a program for calculating X-ray diffuse scattering intensities from molecular dynamics trajectories, Comput. Phys. Commun. 91(1-3), 331-338 (1995).

[24] F. Merzel, and J. C. Smith, SASSIM: a method for calculating small-angle X-ray and neutron scattering and the associated molecular envelope from explicit-atom models of solvated proteins, Acta Crystallographica Section D Biological Crystallography 58, 242-249 (2002).

[25] D. Svergun, C. Barberato, CRYSOL-a program to evaluate X-ray solution scatter-ing of biological macromolecules from atomic coordinates, J. Appl. Crys. 28, 768-773 (1995).

[26] K. Hinsen, E. Pelligrini, S. Stachura, G. R. Kneller, NMoldyn 3: Using task farming for a parallel spectroscopy-oriented analysis of molecular dynamics simulations, J. Comp. Chem. 33, 2043-2048 (2012).

[27] B. Lindner, J. Smith, Sassena - X-ray and neutron scattering calculated from molecular dynamics trajectories using massively parallel computers, Comput. Phys. Commun. 183, 1491 (2012).

[28] W. C. Swope, J. W. Pitera, and F. Suits, Describing Protein Folding Kinetics by Molecular Dynamics Simulations, J. Phys. Chem. B 108, 6571 (2004).

[29] F. Noé, I. Horenko, C. Schütte, and J. C. Smith, Hierarchical analysis of conformational dynamics in biomolecules: Transition networks of metastable states, J. Chem. Phys. 126, 155102 (2007).

[30] B. Keller, J.-H. Prinz, and F. Noé, Markov models and dynamical fingerprints: Unraveling the complexity of molecular kinetics, Chem. Phys. 396, 92 (2012).

[31] F. Noé and S. Fischer, Transition networks for modeling the kinetics of conformational change in macromolecules, Curr. Opin. Struct. Biol. 18, 154 (2008).

[32] V. Pande, K. Beauchamp, and G. Bowman, Everything you wanted to know about Markov State Models but were afraid to ask, Methods 52, 99 (2010). 
[33] F. Noé, S. Doose, I. Daidone, M. Löllmann, J. Chodera, M. Sauer, and J. Smith, Dynamical fingerprints for probing individual relaxation processes in biomolecular dynamics with simulations and kinetic experiments, Prod. Natl. Acad. Sci. U.S.A. 108, 4822 (2011).

[34] B. Lindner, Z. Yi, J.-H. Prinz, J. C. Smith, and F. Noé, Dynamic neutron scattering from conformational dynamics. I. Theory and Markov models, J. Chem. Phys. 139, 175101 (2013).

[35] Z. Yi, B. Lindner, J.-H. Prinz, F. Noé and J. C. Smith, Dynamic neutron scattering from conformational dynamics. II. Application using molecular dynamics simulation and Markov modeling, J. Chem. Phys. 139, 175102 (2013).

[36] L. Hong, N. Smolin, B. Lindner, A. P. Sokolov, and J. C. Smith, Three Classes of Motion in the Dynamic Neutron-Scattering Susceptibility of a Globular Protein, Phys. Rev. Lett. 107, 148102 (2011).

[37] J. A. Rupley and G. Careri, Protein Hydration and Function, Adv. Protein Chem. 41, 37 (1991).

[38] M. Ferrand, A. J. Dianoux, W. Petry, and G. Zaccai, Thermal motions and function of bacteriorhodopsin in purple membranes: effects of temperature and hydration studied by neutron scattering, Proc. Natl. Acad. Sci. U.S.A. 90, 9668 (1993).

[39] A. Tamura, M. Matsushita, A. Naito, S. Kojima, K.-I. Miura, and K. Akasaka, Dynamics of the three methionyl side chains of Streptomyces sub tilisin inhibitor. Deuterium NMR studies in solution and in the solid state, Protein Sci. 5, 127 (1996).

[40] G. Careri and E. Gratton, in The Fluctuating Enzyme, edited by G. R. Welch (Wiley, New York, 1986), p. 239

[41] J. M. Zanotti, M. C. Bellissent-Funel, and J. Parello, Hydration-coupled dynamics in proteins studied by neutron scattering and NMR: the case of the typical EF-hand calcium-binding parvalbumin, Biophys. J. 76, 2390 (1999).

[42] E. Kussell and E. I. Shakhnovich, Glassy Dynamics off Side-Chain Ordering in a Simple Model of Protein Folding, Phys. Rev. Lett. 89, 168101 (2002).

[43] See supplemental material at http://link.aps.org/supplemental/10.1103/PhysRevLett.108.238102 for details of the MD protocol and analytic procedure.

[44] A. Kitao, F. Hirata, and N. Go, The effect of solvent on the conformation and the collective motions of protein: Normal mode analysis and molecular dynamics simulations of melittin in water and in vacuum, Chem. Phys. 158, 447 (1991).

[45] L. Hong, X. Cheng, D. C. Glass, J. D. Nickels, and J. C. Smith, Surface Hydration Amplifies SingleWell Protein Atom Difffusion Propogating into the Macromolecular Core, Phys. Rev. Lett. 108, 238102 (2012).

[46] L. Hong, D. C. Glass, J. D. Nickels, S. Perticaroli, Z. Yi, Z., T. Madhusudan, H. O'Neill, Q. Zhang, A. P. Sokolov, and J. C. Smith, Elastic and Conformational Softness of a Globular Protein, Phys. Rev. Lett. 110, 028104 (2013).

[47] R. Inoue, R. Biehl, T. Rosenkranz, J. Fitter, M. Monkenbusch, A. Radulescu, B. Farago, and D. Richter, Large Domain Fluctuations on 50-ns Timescale Enable Catalytic Activity in Phosphoglycerate Kinase, Biophys. J. 99, 2309 (2010). 
[48] N. Smolin, R. Biehl, G. R. Kneller, D. Richter, and J. C. Smith, Functional Domain Motions in Proteins on the 1-100 ns Timescale: Comparison of Neutron Spin-Echo Spectroscopy of Phosphoglycerate Kinase with Molecular-Dynamics Simulation, Biophys. J. 102, 1108 (2012).

[49] Z. M. Bu, R. Biehl, M. Monkenbusch, D. Richter, and D. J. E. Callaway, Coupled protein domain motion in Taq polymerase revealed by neutron spin-echo spectroscopy, Proc. Natl. Acad. Sci. U.S.A. 102, 17646-17651 (2005).

[50] B. Farago, J. Li, G. Cornilescu, D. J. E. Callaway, and Z. Bu, Activation of Nanoscale Allosteric Protein Domain Motion Revealed by Neutron Spin Echo Spectroscopy, Biophys. J. 99, 3473 (2010).

[51] K. Skold, Smallenergy transfer scattering of cold neutrons from liquid argon, Phys. Rev. Lett. 19, 1023 (1967).

[52] G. Venkatar, Ba. Dasannac, and K. R. Rao, Cold neutron scatterin from liquid $\mathrm{CD}_{4}$, Phys. Lett. 23, 226 (1966).

[53] J. C. Brown, P. N. Pusey, J.W. Goodwin, and R. H. Ottewill, Light scattering study of dynamcisand time-averaged correlations in dispersions of charged particles, J. Phys. A 8, 664 (1975).

[54] D.W. Schaefer and B. J. Berne, Dynamics of Charged Macromolecules in Solution, Phys. Rev. Lett. 32, 1110 (1974).

[55] J. K. Phalakornkul, A. Gast, R. Pecora, G. Nägele, A. Ferrante, B. Mandl-Steininger, and R. Klein, Structure and short-time dynamics of polydisperse charge-stabilized suspension, Phys. Rev. E 54, 661 (1996).

[56] P. G. de Gennes, Liquid dynamics and inelastic scattering of neutrons, Physica (Amsterdam) 25, 825 (1959).

[57] B. J. Ackerson, P. N. Pusey, and R. J. A. Tough, Interpretation of the intermediate scattering function at short times, J. Chem. Phys. 76, 1279 (1982).

[58] L. Hong, N. Simolin, and J. C. Smith, de Gennes Narrowing Describes the Relative Motion of Protein Domains, Phys. Rev. Lett. 112, 158102 (2014).

[59] H. C. Watson, N. P. Walker, P. J. Shaw, T. N. Bryant, P. L. Wendell, L. A. Fothergill, R. E. Perkins, S. C. Conroy, M. J. Dobson, and M. F. Tuite) Sequence and structure of yeast phosphoglycerate kinase, The EMBO Journal 1, 1635-1640 (1982).

[60] K. Henzler-Wildman, and D. Kern, Dynamic personalities of proteins. Nature 450, 964-972 (2007).

[61] Y. Miao, Z. Yi, C. Cantrell, D. C. Glass, J. Baudry, N. Jain, and J. C. Smith, Coupled Flexibility Change in Cytochrome P450cam Substrate Binding Determined by Neutron Scattering, NMR, and Molecular Dynamics Simulation, Biophys. J. 103(10), 2167-2176 (2012).

[62] T. L. Poulos, Cytochrome P450 flexibility. PNAS. 100, 13121-13122 (2003).

[63] E. Mamontov and K. W. Herwig, A time-of-flight backscattering spectrometer at the Spallation Neutron Source, BASIS, Rev. Sci. Inst. 82(8), 085109 (2011).

[64] G. Ehlers, A. A. Podlesnyak, and P. E. Sokol, The new cold neutron chopper spectrometer at the pallation Neutron Source: design and performance. Rev. Sci. Instrum. 2011;82:085108. 
[65] G. Lipari, and A. Szabo, Model-free approach to the interpretation of nuclear magnetic resonance relaxation in macromolecules. 1. Theory and range of validity. J. Am. Chem. Soc. 104, 4546-4549 (1982).

[66] G. Lipari, and A. Szabo, Model-free approach to the interpretation of nuclear magnetic resonance relaxation in macromolecules. 2. Analysis of experimental results. J. Am. Chem. Soc. 104, 4559-4570 (1982).

[67] W. Doster, S. Cusack, S. and W. Petry, W., Dynamical transition of myoglobin revealed by inelastic neutron scattering, Nature 337, 754 (1989).

[68] F. Gabel, D. Bicout, U. Lehnert, M. Tehei, M. Weik, and G. Zaccai, Protein dynamics studied by neutron scattering, Q. Rev. Biophys. 35, 327 (2002).

[69] S. Magaz`u, F. Migliardo, and A. Benedetto, A., Mean square displacement from elastic incoherent neutron scattering evaluated by spectrometers working with different energy resolution on dry and hydrated $\left(\mathrm{H}_{2} \mathrm{O}\right.$ and $\left.\mathrm{D}_{2} \mathrm{O}\right)$ lysozyme, J. Phys. Chem. B 114, 9268 (2010).

[70] S. Magaz`u, F. Migliardo, and A. Benedetto, A., Puzzle of protein dynamical transition, J. Phys. Chem. B 115, 7736 (2011).

[71] A. L. Tournier and J. C. Smith, Principal Components of the Protein Dynamical Transition, Phys. Rev. Lett. 91, 208106 (2003).

[72] Y. L. Miao, Z. Yi, D. C. Glass, L. Hong, M. Tyagi, J. Baudry, N. Jain, and J. C. Smith, TemperatureDependent Dynamical Transitions of Different Classes of Amino Acid Residue in a Globular Protein, Journal of the American Chemical Society 134(48), 19576-19579 (2012).

[73] K. Wood, S. Grudinin, B. Kessler, M. Weik, M. Johnson, G. R. Kneller, D. Oesterhelt, and G. Zaccai, Dynamical heterogeneity of specific amino acids in bacteriorhodopsin, J. Mol. Biol. 380, 581 (2008).

[74] M. Jasnin, L. van Eijck, M. M. Koza, J. Peters, C. Laguri, H. Lortat-Jacob and G. Zaccai, Dynamics of heparan sulfate explored by neutron scattering, Phys. Chem. Chem. Phys. 12, 3360 (2010).

[75] H. Nakagawa, H. Kamikubo, M. Kataoka, Effect of conformational states on protein dynamical transition, Biochim. Biophys. Acta 1804, 27 (2010).

[76] D. Vural, and H. R. Glyde, Intrinsic mean-square displacements in proteins, Phys. Rev. E 86, 011926 (2012).

[77] D. Vural, L. Hong, J. C. Smith and H. R. Glyde, Long-time mean-square displacement in proteins, Phys. Rev. E 88, 052706 (2013).

[78] R. M. Daniel, R. V. Dunn, J. L. Finney, and J. C. Smith, The role of dynamics in enzyme activity, Annu. Rev. Biophys. Biomol. Struct. 32, 69 (2003).

[79] G. Schiro, F. Natali, andA.Cupane, Physical origin of anharmonic dynamics in proteins: new insights from resolution-dependent neutron scattering on homomeric polypeptides, Phys. Rev. Lett. 109, 128102 (2012).

[80] V. Calandrini, V. Hamon, K. Hinsen, P. Calligari, M.-C. Bellissent-Funel, and G. R. Kneller, Relaxation dynamics of lysozyme in solution under pressure: Combining molecular dynamics simulations and quasielastic neutron scattering, Chem. Phys. 345, 289 (2008). 
[81] D. Vural, L. Hong, J. C. Smith and H. R. Glyde, Motional displacement in proteins: The origin of wave-vector-dependent values, Phys. Rev. E 91, 052705 (2015).

[82] H. Yang, G. Luo, P. Karnchanaphanurach, T.-M. Louie, I. Rech, S. Cova, L. Xun and X. S. Xie,, Protein conformational dynamics probedby single-molecule electron transfer, Science 320, 262-266 (2003).

[83] W. Min, G. Luo, B. J. Cherayil, S. C. Kou and X. S. Xie, Observation of a Power-Law Memory Kernel for Fluctuations within a single protein molecules, Phys. Rev.Lett. 94, 198302 (2005).

[84] X. Hu, L. Hong, M. D. Smith, T. Neusius, X. Cheng and J. C. Smith, The dynamics of single protein molecules is non-equilibrium and self-similar over thirteen decades in time, Nat. Phys. 12, 171 (2016).

[85] S. Burov R. Metzler and E. Barkai, Aging and nonergodicity beyond the Khinchin theorem, PNAS. 107(30), 13228-13233 (2010).

[86] H. Yang, G. Luo, P. Karnchanaphanurach, T. - M. Louie, I. Rech, S. Cova, L. Xun, and X. S. Xie. Protein conformational dynamics probed by single-molecule electron transfer. Science 302, 262 (2003).

[87] A. G. Zilman and R. Granek, Undulations and Dynamic Structure Factor of Membranes, Phys. Rev. Lett. 77, 4788 (1996)

[88] J. Nickels, X.Chen, B. Mostofian, C. Stanley, B. Lindner, F. Heberle, S. Perticaroli, M. Feygenson, T. Egami, R. Standaert, J. Smith, D. Myles, M. Ohl and J. Katsaras. Mechanical properties of nanoscopic lipid domains reveal a mechanism for lipid raft formation, J. Am. Chem. Soc. (2015) in press DOI: 10.1021/jacs.5b08894

[89] Z. A. Levine, R. M. Venable, M. C. Watson, M. G. Lerner, J.-E. Shea, R. W. Pastor, F. L. H. Brown. Determination of biomembrane bending moduli in fully atomistic simulations. J. Am. Chem. Soc. 136, 13582-13585 (2014).

[90] L. Rusevich, V. S. Garcia, B. Franzetti, M. Johnson, F. Natali, E. Pellegrini, J. Peters, J. Pieper, M. Weik, and G. Zaccai, Perspectives in biological physics: the NDDB project for a neutron Dynamics Data Bank for biological macromolecules, Eur. Phys. J. E- Soft Matter 36(7), 1-9 (2013) 